\title{
Adlayer Structure Dependent Ultrafast Desorption Dynamics in Carbon Monoxide Adsorbed on Pd (111)
}

\author{
Sung-Young Hong, Pan Xu, Nina R. Camillone, \\ Michael G. White, and Nicholas Camillone III
}

Submitted to Journal of Chemical Physics

July 2016

Chemistry Department

Brookhaven National Laboratory

\section{U.S. Department of Energy USDOE Office of Science (SC), Basic Energy Sciences (BES) (SC-22)}




\section{DISCLAIMER}

This report was prepared as an account of work sponsored by an agency of the United States Government. Neither the United States Government nor any agency thereof, nor any of their employees, nor any of their contractors, subcontractors, or their employees, makes any warranty, express or implied, or assumes any legal liability or responsibility for the accuracy, completeness, or any third party's use or the results of such use of any information, apparatus, product, or process disclosed, or represents that its use would not infringe privately owned rights. Reference herein to any specific commercial product, process, or service by trade name, trademark, manufacturer, or otherwise, does not necessarily constitute or imply its endorsement, recommendation, or favoring by the United States Government or any agency thereof or its contractors or subcontractors. The views and opinions of authors expressed herein do not necessarily state or reflect those of the United States Government or any agency thereof. 


\title{
Adlayer Structure Dependent Ultrafast Desorption Dynamics in Carbon Monoxide Adsorbed on Pd (111)
}

\author{
Sung-Young Hong, ${ }^{\mathrm{a}}$ Pan Xu, ${ }^{\mathrm{b}}$ Nina R. Camillone, ${ }^{\mathrm{a}}$ Michael G. White, ${ }^{\mathrm{a}, \mathrm{b}}$ \\ and Nicholas Camillone III $^{\mathrm{a}, *}$ \\ ${ }^{a}$ Chemistry Department, Brookhaven National Laboratory, Upton, NY 11973 \\ ${ }^{b}$ Department of Chemistry, Stony Brook University, Stony Brook, NY 11794
}

\begin{abstract}
We report our ultrafast photoinduced desorption investigation of the coverage dependence of substrate-adsorbate energy transfer in carbon monoxide adlayers on the (111) surface of palladium. As the CO coverage is increased, the adsorption site population shifts from all threefold hollow (up to $0.33 \mathrm{ML}$ ), to bridge and near bridge (> 0.5-0.6 ML) and finally to mixed threefold hollow plus top site (at saturation at $0.75 \mathrm{ML}$ ). We show that between 0.24 and $0.75 \mathrm{ML}$ this progression of binding site motifs is accompanied by two remarkable features in the ultrafast photoinduced desorption of the adsorbates: (i) the desorption probability increases roughly two orders magnitude, and (ii) the adsorbate-substrate energy transfer rate observed in two-pulse correlation experiments varies nonmonotonically, having a minimum at intermediate coverages. Simulations using a phenomenological model to describe the adsorbate-substrate energy transfer in terms of frictional coupling indicate that these features are consistent with an adsorption-site dependent electron-mediated energy coupling strength, $\eta_{\mathrm{el}}$, that decreases with binding site in the order: three-fold hollow > bridge and near bridge $>$ top site. This weakening of $\eta_{\text {el }}$ largely counterbalances the decrease in the desorption activation energy that accompanies this progression of adsorption site motifs, moderating what would otherwise be a rise of several orders of magnitude in the desorption probability. Within this framework, the observed energy transfer rate enhancement at saturation coverage is due to interadsorbate energy transfer from the copopulation of molecules bound in three-fold hollows to their top-site neighbors.
\end{abstract}




\section{Introduction}

Molecule-surface energy transfer is central to the surface chemical dynamics of adsorption, desorption, and reaction, and much experimental and theoretical effort has been invested in understanding its mechanisms. Beginning in the 1980s, experiments ${ }^{1-4}$ and theory ${ }^{5,6}$ have demonstrated that electron-hole pairs (ehps) mediate the adsorbate-surface energy transfer responsible for the ultrafast ( ps) relaxation of high frequency ( $>2$ times the substrate Debye frequency ${ }^{7,8}$ ) molecular vibrations at metal surfaces. As a result, the role of ehp-mediated molecule-surface energy coupling more broadly in chemical reaction dynamics at metal surfaces has become the subject of a growing number of studies. ${ }^{9-15}$ Ehp-mediated energy transfer is of interest from a theoretical perspective because it signals nonadiabatic behavior, i.e., the breakdown of the Born-Oppenheimer approximation, ${ }^{16,17}$ and from an experimental perspective because ehp photoexcitation with short ( $\sim 100-\mathrm{fs})$ laser pulses can be used to initiate molecular motion, enabling time-resolved investigations of surface chemical dynamics. ${ }^{13,15}$

Specifically, excitation of an adsorbate-covered metal surface with intense $\left(>\sim 1 \mathrm{~mJ} / \mathrm{cm}^{2}\right)$ short laser pulses can drive diffusion, ${ }^{18,19}$ desorption, ${ }^{20-23}$ and interadsorbate reactions. ${ }^{24-28}$ In such experiments, electrons and phonons may mediate the metal-adsorbate energy transfer. By performing two-pulse correlation (2PC) ${ }^{21}$ or pump-probe experiments, ${ }^{18,29,30}$ the progress of the system along the diffusion, desorption or reaction coordinate can be tracked in real time, and the dominant energy-transfer mechanism(s) and rates inferred. When excited by an ultrafast pulse, the substrate electrons are driven out of thermal equilibrium with the lattice degrees of freedom for $\sim 1 \mathrm{ps}$, and reach temperatures typically in the $2000-7000 \mathrm{~K}$ range. This disequilibrium provides the possibility of distinguishing between electron- and phonon-mediated energy transfer, and the 
high electronic temperatures generally result in the ehp-adsorbate coupling dominating the adsorbate heating that drives the surface chemistry.

To date a number of model systems consisting of various adsorbates $\left(\mathrm{H}_{2}, \mathrm{O}_{2}, \mathrm{O}, \mathrm{CO}, \mathrm{NO}\right.$, $\mathrm{NH}_{3}$ and benzene) on several single-crystal metal (Cu, Ru, Pd, and Pt) surfaces have been studied. ${ }^{2}$, 4, 18-30 As the number of material systems investigated has grown, correlations between the adlayer/substrate electronic structure and the coupling strength have been noted. Specifically, empirically it is becoming clear that the ehp-adsorbate coupling strength increases with the degree of overlap between substrate and adsorbate electronic energy levels, as predicted by early theory. ${ }^{5}$ For example, weaker ehp-adsorbate coupling, and therefore slower desorption dynamics, in $\mathrm{CO} / \mathrm{Ru}(001)$ as compared to $\mathrm{CO} / \mathrm{Cu}(100)$ was attributed to the $\mathrm{CO} 2 \pi^{*}$ lying $2 \mathrm{eV}$ higher above the Fermi level of Ru than $\mathrm{Cu}^{23}$ The higher the adsorbate affinity energy level, the less overlap with the photoexcited electron distribution, and therefore the weaker the coupling. In previous work we have also suggested that coupling strength may be linked to spatial overlap: we observed a decrease in ehp-adsorbate coupling strength with increasing coverage in $\mathrm{O}_{2} / \mathrm{Pd}(111)^{31}$ that correlated with an increase in adsorbate-substrate bond length. ${ }^{32}$ The longer the adsorbatesubstrate bond, the less orbital spatial overlap, the weaker the nonadiabatic frictional forces ${ }^{6}$ and lower the probability of interfacial electron transfer. ${ }^{33}$ More recently, a time-resolved study of transient $\mathrm{C}-\mathrm{O}$ stretch frequency shifts on $\mathrm{Cu}(110)$ induced by short-pulse excitation of the substrate indicated that the degree of nonadiabaticity increases with coverage. ${ }^{34} \mathrm{For} \mathrm{CO} / \mathrm{Cu}(110)$, the overlap of the $2 \pi^{*}$ with the copper d-band increases with increasing coverage, as evidenced by a negative "chemical shift" in the C-O stretch frequency. ${ }^{35}$ Thus, this work harmonizes well with the emerging picture that ehp coupling strength correlates with the degree of orbital overlap. 
The work described herein explores correlations between nonadiabaticity and adlayer structure using femtosecond laser photoinduced desorption (fs-PID). Specifically, we investigate the coverage dependence of substrate-adsorbate energy coupling with a well-characterized model system, $\mathrm{CO} / \mathrm{Pd}(111)$, that offers the possibility of extracting the adsorption-site dependence of the coupling. This system is well-known for its structural complexity, exhibiting at least 17 ordered adlayer structures. ${ }^{36}$ Infrared reflection-absorption ${ }^{37,} 38$ and sum-frequency generation ${ }^{39}$ spectroscopies, low-energy electron diffraction (LEED), ${ }^{36,40,41}$ photoelectron diffraction, ${ }^{42}$ highresolution $x$-ray photoelectron spectroscopy, ${ }^{43}$ scanning tunneling microscopy ${ }^{44}$ and computational studies ${ }^{45-47}$ together show a progression of binding motifs: exclusively three-fold hollow (3-fh) sites at or below 0.33ML, mixed 3-fh and bridge sites at 0.5 ML, predominantly bridge and near-bridge sites between 0.5 and $0.67 \mathrm{ML},{ }^{36}$ with top sites and 3fh sites possibly occupied above 0.6-ML, ${ }^{36}$ until ultimately, a mixed 3-fh and top-site structure develops near saturation at $0.75 \mathrm{ML}$. By controlling the coverage it is therefore possible to determine, to some extent, the binding site from which the adsorbate is initially excited in a fs-PID experiment.

Density functional theory (DFT) calculations show that the $2 \pi^{*}$ orbital fractional occupation — a proxy for the degree of the $2 \pi^{*}$ overlap with the Pd d-bands—decreases in the order: $3 \mathrm{fh}>$ bridge $>$ top. ${ }^{48}$ This trend correlates well with calculated and experimental trends across this progression of binding motifs: the adsorption energy decreases, the C-surface distance increases, the $\mathrm{C}-\mathrm{O}$ bond length decreases toward the gas-phase value and the $\mathrm{C}-\mathrm{O}$ stretch frequency blue shifts toward the gas-phase value. Thus, by all indications we would anticipate a decrease in the $\mathrm{Pd}-\mathrm{CO}$ coupling strength with increasing coverage.

Our measurements show a dramatic dependence of the fs-PID probability on coverage across a broad coverage range (0.1-0.75 ML). Of particular note is a $~ 100$-fold increase between 
$\sim 0.3$ and $0.75 \mathrm{ML}$. While this might seem counter to the aforementioned trend in coupling strength, we show that simulations using a commonly employed phenomenological model to describe the adsorbate-substrate energy transfer in terms of a frictional coupling ${ }^{19,23,49-52}$ indicate that though the bridge site $\mathrm{CO}$ are more weakly coupled to the substrate than the $3 \mathrm{fh} \mathrm{CO}$, their desorption probability is much higher because the weak coupling is more than offset by the lower desorption activation energy.

However, the story is not as straightforward as a simple partial compensation of the decrease in desorption activation energy by the decrease in coupling strength with increasing coverage. Instead our time-resolved measurements of three well-defined structures (Figure 1) spanning the $0.24-0.75-\mathrm{ML}$ range show that the 100 -fold increase in fs-PID probability is accompanied by a nonmonotonically varying coupling time. As expected, coupling is fast at the lowest coverage, where only the 3fh site is occupied, and slower at higher coverage where bridge and near-bridge sites are occupied. However, the coupling is faster at $0.75 \mathrm{ML}$, where the top sites are occupied, than for the intermediate, bridgesite population, coverage. We suggest a simple explanation: the top-site CO are most weakly coupled to the Pd, however, because the packing is so dense at 0.75 ML, energy is efficiently transferred to top-site CO from the strongly-coupled copopulation of 3fh CO.

(a)

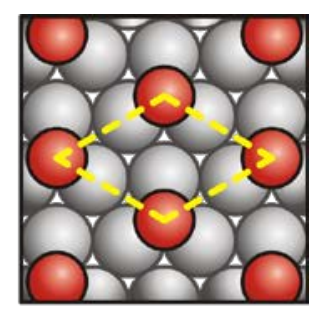

(b)

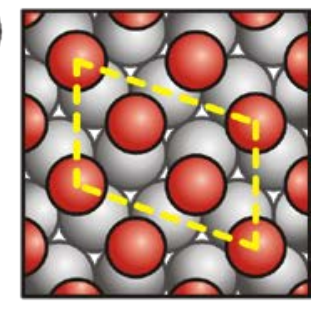

(c)
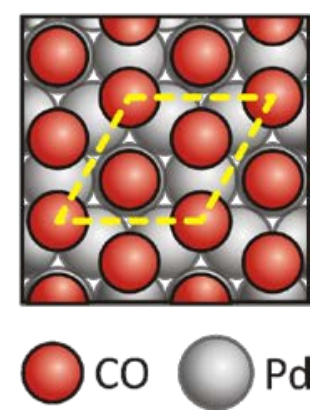

Figure 1. Coverage dependence of the CO adlayer structure on $\mathrm{Pd}(111)$ : (a) $0.33 \mathrm{ML}$ $(\sqrt{3} \times \sqrt{3}) \mathrm{R} 30^{\circ}$, (b) $0.60 \mathrm{ML} \mathrm{c}(\sqrt{3} \times 5)$, and (c) $0.75 \mathrm{ML}(2 \times 2)$. Dotted yellow lines indicate the unit mesh. 
Thus, we conclude that the electron-mediated energy coupling strength decreases with binding site in the order: $3 f$ h $>$ bridge $>$ top site, and that interadsorbate energy transfer mediates the energy transfer to the top-site $\mathrm{CO}$ at the highest coverages. A simple model that captures the essence of the coverage-dependent behavior between 0.24 and $0.75 \mathrm{ML}$ is developed.

\section{Materials and Methods}

All the experiments were conducted in an ultrahigh vacuum (UHV) chamber ( $p<10^{-10}$ Torr) equipped with a liquid- $\mathrm{N}_{2}$-cooled sample manipulator, a sputter gun, a low-energy electron diffractometer, an Auger spectrometer, two retractable pinhole $(\sim 5 \mu \mathrm{m})$ gas dosers and a quadruple mass spectrometer (QMS). Other experimental details have been published previously. ${ }^{31,50}$

Initial preparation of the $\mathrm{Pd}(111)$ involved cycles of sputtering $\left(\mathrm{Ar}^{+}, 700 \mathrm{eV}, 1.2 \mu \mathrm{A}\right.$, $20 \mathrm{~min})$, annealing $(1200 \mathrm{~K})$ and oxidation (700-1100 K). For daily cleaning, we found that oxidation under $\mathrm{O}_{2}\left(p \sim 10^{-8}\right.$ Torr) during cycling between 770 and $1100 \mathrm{~K}$ at $2 \mathrm{~K} / \mathrm{s}$ followed by flashing to $1000 \mathrm{~K}$ resulted in high-quality surfaces as judged by: (i) a sharp $(1 \times 1)$ LEED pattern, (ii) the absence of $\mathrm{CO}_{2}$ and $\mathrm{CO}$ signals below $\sim 800 \mathrm{~K}$ in temperature-programmed desorption (TPD) measurements following oxygen exposure, and (iii) a sharp $(2 \times 2)$ LEED pattern at saturation CO coverage.

${ }^{12} \mathrm{C}^{18} \mathrm{O}$ was used in all experiments to avoid the ${ }^{12} \mathrm{C}^{16} \mathrm{O}$ background. CO coverage was precisely and reproducibly metered by controlling the exposure time, pressure behind the doser pinhole, and annealing temperature. The three coverages for which extensive fluence-dependence and time-resolved correlation measurements were made were prepared as follows: (i) $(\sqrt{3} \times$ $\sqrt{3}) R 30^{\circ} \mathrm{CO}$ - exposure to $\mathrm{CO}$ at $210 \mathrm{~K}$ followed by a 2-min anneal at $280 \mathrm{~K}$; (ii) $\mathrm{c}(\sqrt{3} \times 5)$ 
CO—exposure at $135 \mathrm{~K}$ followed by a 10-s anneal at $220 \mathrm{~K}$; (iii) $(2 \times 2) \mathrm{CO}$ —exposure at $135 \mathrm{~K}$ until saturation. In addition, for the survey of fs-PID probabilities coverages in the $0.05-0.33-\mathrm{ML}$ range were prepared by varying the $\mathrm{CO}$ exposure time at $210 \mathrm{~K}$ with annealing to $280 \mathrm{~K}$, and in the $0.33-0.75-\mathrm{ML}$ range by varying the exposure time and temperature. Saturation was judged by the observation of a well-developed low-temperature feature at $~ 165 \mathrm{~K}$ in the TPD and a sharp $(2 \times 2)$ LEED pattern (see supplemental material, Figure S1 $c^{53}$ ). The growth of well-ordered $(\sqrt{3} \times \sqrt{3}) R 30^{\circ}$ and $\mathrm{c}(\sqrt{3} \times 5)$ adlayer structures were confirmed by the observation of highquality LEED patterns (see supplemental material, Figures S1a and S1b ${ }^{53}$ ), and uniformity of coverage across the sample for all coverages was confirmed by LEED as well. For the three preparations, the experimentally-determined coverages were 0.24 ML, 0.64 ML, and 0.75 ML, based on the ratio of the temperature-integrated TPD yields to that measured at saturation, assuming a saturation coverage of $0.75 \mathrm{ML}$. These coverage numbers are the ones cited throughout the paper. They are somewhat different from the ideal coverages, $0.33,0.60$ and $0.75 \mathrm{ML}$ (Figure 1) for two reasons. First, there is some experimental uncertainty due to spurious desorption associated with adsorption on the manipulator during saturation exposures. Second, after careful analysis we found that $0.24 \mathrm{ML}$ was, in fact, somewhat lower than the highest coverage at which a clean $(\sqrt{3} \times \sqrt{3}) R 30^{\circ}$ LEED is observed. Nevertheless, our LEED observations were consistent with STM work ${ }^{44}$ that showed well-developed $(\sqrt{3} \times \sqrt{3}) R 30^{\circ}$ islands at coverages below $0.33 \mathrm{ML}$, and the coverage dependence of the fs-PID in the 0.2-0.4-ML range was found to be comparatively weak (vide infra).

The light source for the fs-PID experiments was a regenerative amplifier generating 4-mJ, 100-fs, $780-\mathrm{nm}$ pulses, operated at a repetition rate of $8 \mathrm{~Hz}$. The pulse energy delivered to the surface was controlled by a variable attenuator composed of a zero-order half-wave plate and a 
pair of thin-film polarizers. The light was focused so as to achieve a spot diameter of $\sim 0.6 \mathrm{~mm}$ at the surface, as measured by a CCD camera fixed at a conjugate distance from a beam sampler outside the UHV system. Based on the spot profile and the measured reflected energy, the peak absorbed fluence was controlled to be in the range of $5-14 \mathrm{~mJ} / \mathrm{cm}^{2}$.

For 2PC measurements the pulses were split into two orthogonally-polarized components. The component pulses traversed a modified Mach-Zehnder interferometer wherein the length of one of the arms was varied by computer-controlled translation of a hollow retroreflector. The delay between the component pulses was defined as positive when the weaker, s-polarized pulse arrived before the stronger, p-polarized one. The pulses were directed non-collinearly to the Pd surface, separated by an angle of $\sim 0.1^{\circ}$ to minimize optical interference near zero delay that resulted from slight depolarization in the UHV window. The nominal incident angle was $50^{\circ}$ relative to the surface normal. The pulse width at the Pd surface (130-fs full-width at half-maximum, sech ${ }^{2}$ ) and the zero-delay retroreflector position were determined by in situ surface second harmonic generation cross-correlation at the Pd surface.

All the fs-PID measurements were conducted at a substrate temperature of $90 \mathrm{~K}$. The number of pulses admitted to the chamber was controlled by a computer-triggered electromechanical shutter synced to the laser pulse train. Both the desorption yield (measured by the QMS in pulse-counting mode) and the reflected energy were recorded on a shot-to-shot basis. Typically, anywhere between 10 and 400 shots were recorded, to simply measure the first-shot yield or follow the depletion of the adlayer in detail, respectively. By translating the Pd across the laser, up to 25 measurements could be made over the face of the surface of a single adlayer preparation. Subsequently, the surface was cleaned by TPD up to $700 \mathrm{~K}$, and a new adlayer prepared. 
To compare simulations to measurements, it is important to make a reasonable estimate of the absolute desorption probability. We have done this by determining the overall sensitivity of our setup such that the desorption probability can be estimated from a single-shot measurement of the desorption yield (in QMS counts) and the measured laser spot profile. We begin by expressing the desorption yield (in QMS counts), $Y_{S}$, for $S^{\text {th }}$ shot as

$$
Y_{S}=N \sigma F_{\text {peak }}^{n} \iint \theta_{S-1}(x, y) f(x, y)^{n} d x d y
$$

where $N$ is an instrumental scaling factor that converts the number of desorbing molecules to the measured counts, $\sigma$ is the photodesorption cross section, $F_{\text {peak }}$ is the peak absorbed fluence, $n$ is the power-law exponent characteristic of the nonlinear dependence of the yield on the laser fluence, $\theta_{S}(x, y)$ is the coverage remaining following desorption by the $S^{\text {th }}$ pulse, and $f(x, y)$ is the amplitude-normalized laser spot spatial distribution. Except for $\sigma$ and $N$, all of the parameters are experimentally determined (measured beam profile, absorbed pulse energy, fluence dependence of the yield, initial coverage). Assuming $\sigma, n$, and $N$ to be constant, $\sigma$ and $N$ are determined from a best fit of Equation 1 to the yield measured as function of shot extended towards full depletion of the adlayer. The resultant scaling factor can then be used to determine $\sigma$ and, thereby, the first-shot desorption probability at the laser spot profile peak $\left(P_{1, p e a k}\right)$, according to

$$
\begin{gathered}
Y_{1}=N \sigma F_{\text {peak }}^{n} \theta_{0} \iint f(x, y)^{n} d x d y \text { and } \\
P_{1, \text { peak }}=\sigma F_{\text {peak }}^{n},
\end{gathered}
$$

from measured first-shot yields, $Y_{1}$, laser spot profiles and absorbed pulse energies in subsequent experiments. 


\section{Experimental Results}

Figure 2 shows the fluence dependence of the first-shot fs-PID probability for CO from $\mathrm{CO} / \mathrm{Pd}(111)$ at three selected initial coverages. The measured fs-PID yields have been converted to probabilities for display in Figure 2 using Equations 2 and 3: For each data point, a cross section, $\sigma$, was computed from the measured firstshot yield (QMS counts) using Equation 2, given the scaling factor, $N$, the initial coverage, $\theta_{0}$, the spatial profile, $f(x, y)$, peak fluence, $F_{\text {peak }}^{n}$, and power-law exponent, $n$. With the thus computed

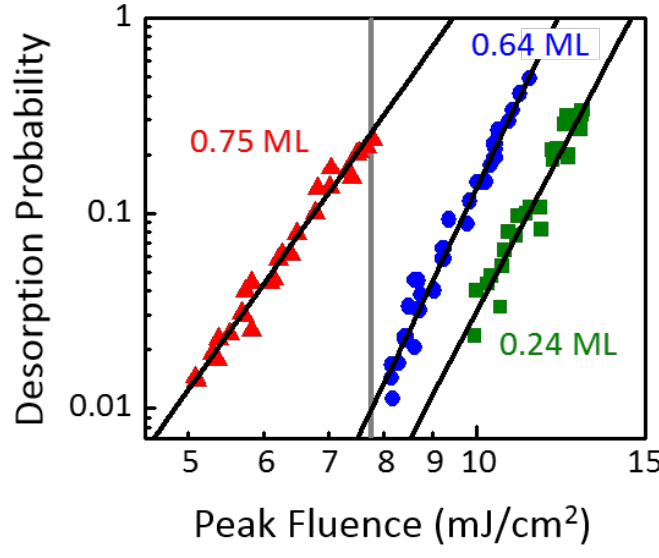

Figure 2. The fluence dependence of the fs-PID probability for $\mathrm{CO}$ from $\mathrm{CO} / \mathrm{Pd}(111)$ at 0.24 (squares), 0.64 (circles), $0.75 \mathrm{ML}$ (triangles). The solid lines are linear fits to the data, giving slopes of 9.3, 10.3 and 6.9 , respectively. The vertical line marks the $7.76 \mathrm{~mJ} / \mathrm{cm}^{2}$ reference fluence used for the coverage dependence in Figure 3.

cross section, Equation 3 was used to compute the desorption probability at the peak fluence of the laser spot. Because $n$ is not known a priori, the application of Equations 2 and 3 was performed iteratively until a self-consistent value for $n$ was reached, with the initial value taken as the slope of a log-log plot of the raw desorption yield (counts) versus absorbed pulse energy (mJ). At high fluences we observed that the desorption probabilities fall below that expected from this powerlaw behavior. This drop off is due to the fact that once the fluence in the central high-fluence portion of the laser spot is sufficient to completely deplete the surface of adsorbates, the yield cannot continue to increase with fluence at the same rate. These data are not shown in Figure 2 and were excluded from the fit and the determination of $n$.

For initial coverages, $\theta_{0}$, equal to $0.24,0.64$ and $0.75 \mathrm{ML}$, we find $n=9.3 \pm 0.5,10.3 \pm 0.4$ and $6.9 \pm 0.3$, respectively. Within the conventional model for fs-PID, this strong nonlinearity 
stems from the fact that the desorption is an activated process where the barrier-crossing probability depends exponentially on the adsorbate temperature. The fluence dependence of the fs-PID probability for $\mathrm{CO}$ from $\mathrm{CO} / \mathrm{Pd}(111)$ is no exception to this behavior, and value of $n$ we measure at $0.24 \mathrm{ML}$ is within experimental uncertainty of that found in our previous study of CO fs-PID from $(\sqrt{3} \times \sqrt{3}) R 30^{\circ} \mathrm{CO} / \mathrm{Pd}(111) .^{54}$

Figure 3 shows the results of measurements of the first-shot fs-PID probability for CO from

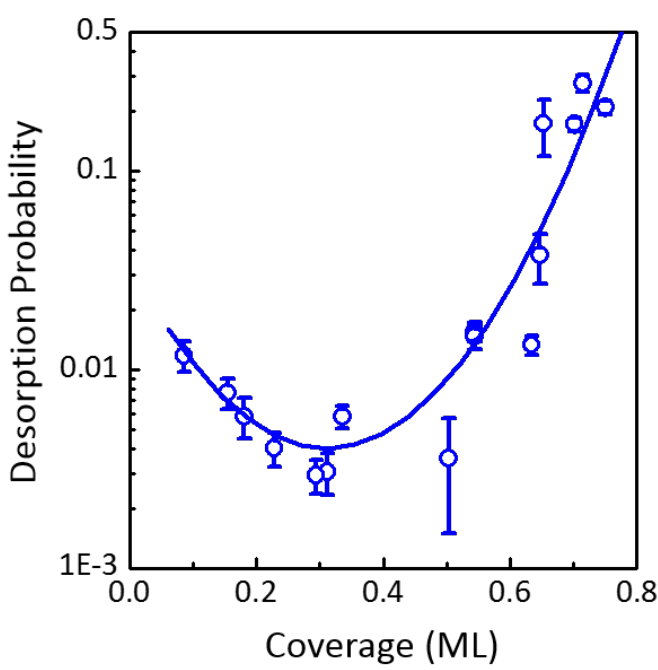

Figure 3. The fs-PID probability for CO from $\mathrm{CO} / \mathrm{Pd}(111)$ as a function of coverage. The probabilities presented here are extrapolated to those expected for excitation at $7.76 \mathrm{~mJ} / \mathrm{cm}^{2}$ fluence, as described in the text. The line is a thirdorder polynomial fit that serves to guide the eye.

$\mathrm{CO} / \mathrm{Pd}(111)$ at an incident laser fluence of $7.76 \mathrm{~mJ} / \mathrm{cm}^{2}$ as a function of coverage from $<0.1$ to 0.75 ML (saturation). Remarkably, the desorption probability ranges over roughly 2 decades, from a minimum as low as $\sim 0.003$ at $\sim 0.3 \mathrm{ML}$, to nearly 0.3 at $0.75 \mathrm{ML}$. This variation in probability is far larger than any found previously in other systems for which coverage dependent probabilities have been measured, including $\mathrm{O}_{2} / \mathrm{Pd}(111),{ }^{31} \mathrm{CO} / \mathrm{Ru}(001),{ }^{23} \mathrm{CO} / \mathrm{Pt}(111),{ }^{55}$ and $\mathrm{NH}_{3} / \mathrm{Pt}(111),{ }^{56}$ where the maximum variation was a factor of $\sim 3$.

The range of probabilities spanned in Figure 3 is so large that a straightforward measurement of the coverage dependence at a single fluence is problematic. At one extreme, the yield from the 0.3-ML adlayer at a fluence of $\sim 8 \mathrm{~mJ} / \mathrm{cm}^{2}$ is too small to be measured from a 0.6-mm laser spot diameter given the sensitivity of our current setup. At the other extreme, a single $\sim 8 \mathrm{~mJ} / \mathrm{cm}^{2}$ laser pulse is sufficient to desorb the entire population of the most-loosely-bound top-site molecules from the 0.75-ML adlayer. For this reason, the probabilities reported in Figure 
3 were measured in two fluence regimes: high fluences of $\sim 12-14 \mathrm{~mJ} / \mathrm{cm}^{2}$ were used in the low coverage regime, and lower fluences of $\sim 7-8 \mathrm{~mJ} / \mathrm{cm}^{2}$ were used in the high coverage regime. To present the data for comparison across the full coverage range in Figure 3, we extrapolated the probabilities to those expected at a constant fluence of $7.76 \mathrm{~mJ} / \mathrm{cm}^{2}$ from those directly measured at the various fluences dictated by the experimental requirements. This was achieved by computing the cross sections, $\sigma$, using Equation 2, and then extrapolating to a peak fluence of $7.76 \mathrm{~mJ} / \mathrm{cm}^{2}$ using Equation 3. Because $n$ is needed to make this extrapolation, the determination of the fluence dependence of the fs-PID probability as a function of coverage (Figure 2) was a crucial first step to quantifying the coverage dependence of the fs-PID probability (Figure 3).

The fact that the degree of nonlinearity, $n$, depends on the coverage highlights an inherent difficulty in comparing the desorption probabilities at different coverages. Specifically, if $n$ depends on the coverage, then the form of the coverage dependence of the probability (Figure 3) will depend on the value of the fluence at which it is evaluated. We chose $7.76 \mathrm{~mJ} / \mathrm{cm}^{2}$ as the best representative fluence because it is near the high fluence limit for the $0.75 \mathrm{ML}$ adlayer. At higher fluences the desorption probability at the center of the laser spot approaches unity, limiting the measured yield. Thus measuring the coverage dependence of the probability at fluences higher than $\sim 8 \mathrm{~mJ} / \mathrm{cm}^{2}$ would unfairly increase the probabilities in the lower coverage range relative to those at the higher coverages. Alternatively, extrapolating the probabilities in the high coverage range to fluences higher than $\sim 8 \mathrm{~mJ} / \mathrm{cm}^{2}$ using Equation 3 would force us to report unphysical probabilities larger than unity. Thus, $7.76 \mathrm{~mJ} / \mathrm{cm}^{2}$ is close to the maximum fluence at which a fair and meaningful comparison can be made across the full coverage range.

It is important to note that choosing a lower fluence (lower than $7.76 \mathrm{~mJ} / \mathrm{cm}^{2}$ ) at which to evaluate the coverage dependence of the probability, would only result in a more pronounced 
difference between the probabilities at high coverage compared to those at low coverage. This is because the degree of nonlinearity for most coverages is greater than or equal to that found at $0.75 \mathrm{ML}$ (n.b., the values for $n$ at 0.24 and 0.64 are greater than that at 0.75 , see Figure 2). Thus extrapolating the desorption probabilities to fluences less than $7.76 \mathrm{~mJ} / \mathrm{cm}^{2}$ would only serve to decrease the probabilities in the low coverage range relative to those near saturation coverage, amplifying the magnitude of the range of the probabilities spanned by the trend in Figure 3 .

Our survey of the fs-PID probability across a wide range of CO coverages reveals a strong trend worth further investigation. Specifically, we wish to understand the substrate-adsorbate energy transfer and desorption dynamics that underpin the observed coverage dependence. Twopulse correlation measurements provide time-resolved access to those dynamics. A 2PC measurement consists of measurement of the fs-PID probability excited by two pulses as a function of the time delay between them, and the dependence of the desorption probability on the delay is sensitive to the rate of substrate-adsorbate energy transfer and the kinetic parameters that govern the desorption. Because of the strong nonlinearity of the fluence dependence of the desorption probability, the component pulses of a pulse pair that would induce desorption with a high probability $\left(\sim 10^{-1}\right)$ should they arrive together, generally are each individually capable of inducing desorption with nearly negligible probabilities $\left(\sim 10^{-2}-10^{-6}\right.$, depending on $n$ and the ratio of the component pulse energies). Thus the first pulse can be understood as serving to "preheat" the adsorbate-substrate complex while the second pulse provides the excitation needed to drive the desorption with a probability that depends on the system's "memory" of the initial excitation. 
We have employed 2PC, along with the fluence dependence measurements, to investigate the strongest part of the coverage dependence, namely the large increase in the desorption probability between $\sim 0.3$ and $0.75 \mathrm{ML}$. Three 2PC measurements were made at representative CO coverages spanning this range: $0.24,0.64$, and $0.75 \mathrm{ML} \mathrm{CO} / \mathrm{Pd}(111)$. At these coverages, the adlayer meshes, $(\sqrt{3} \times \sqrt{3}) R 30^{\circ}, \mathrm{c}(\sqrt{3} \times 5)$, and $(2 \times 2)$, respectively, are well defined as confirmed by the observation of sharp LEED patterns. In addition, these three coverages represent distinct adsorption-site populations: (i) three-fold hollow, (ii) mixed bridge and near-bridge, and (iii) mixed three-fold hollow and top site, respectively (Figure 1), and thus also afford the opportunity to consider correlating the dynamics with site population.

The two-pulse correlation measurements at 0.24, 0.64, and 0.75 ML are presented in Figures 4 and 5. The total pulse-pair absorbed fluence (see Table I) was adjusted to achieve similar desorption probabilities at zero delay for all coverages and the ratio of the absorbed fluences of the component pulses was held constant at $\sim 2.4: 1$. The asymmetry of the 2PC data with respect to zero delay is expected for pulses of different energies, and provides an additional measure for evaluating the quality of the simulation results described in the next section.

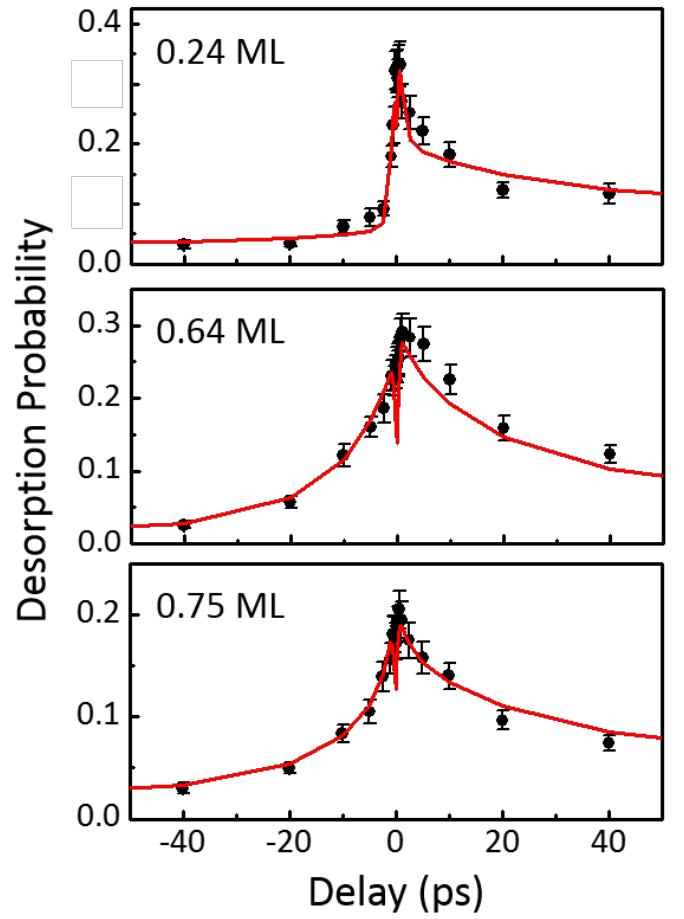

Figure 4. Two pulse correlation (2PC) measurements at initial coverages of $0.24,0.64$ and $0.75 \mathrm{ML}$ (top to bottom). The CO desorption probability was measured as a function of delay between incident p- and s-polarized pulses, where positive delay indicates that the s-polarized pulse arrives first. Pulse fluences are given in Table I. The solid lines are two-temperature model simulations using constant electronic coupling (Model I) as described in the Numerical Modeling section. Only a subset of the full data set extending out to \pm 160 ps delay is shown here. 
Four features in the 2PC data are immediately obvious. First, the widths of the correlations vary with coverage. Table I quantifies the widths of the 2PCs in terms of their half widths at half maximum (HWHM) for both positive $\left(\mathrm{HW}_{+}\right)$and negative $\left(\mathrm{HW}_{-}\right)$sides at each coverage. The HWHM values vary significantly with coverage. The width of a $2 \mathrm{PC}$ measurement reflects the timescale of the substrate-adsorbate energy transfer: the smaller the width, the faster the energy transfer rate, or the stronger the substrate-adsorbate energy coupling. Thus the data indicate that the strength of the substrate-adsorbate coupling depends on coverage, and that nonmonotonically. The implications of this are considered in more detail below.

\section{Table I}

Fluences at the peak of the laser spot profile, for the p-polarized, $F_{\text {peak }}^{\mathrm{p}-\text { pol }}$, and s-polarized, $F_{\text {peak }}^{s-\text { pol }}$, pulses, their sum, $F_{\text {peak }}^{\text {total }}$ and the measured 2PC half widths at half maximum, $\mathrm{HW}_{-}$and $\mathrm{HW}_{+}$for the 2PC measurements for fs-PID of CO from 0.24, 0.64 and $0.75 \mathrm{ML} \mathrm{CO/Pd(111).} \mathrm{Also} \mathrm{given} \mathrm{are} \mathrm{the} \mathrm{power-law}$ exponents for the fluence dependences at the same coverages.

\begin{tabular}{ccccccc}
\hline $\begin{array}{c}\text { Coverage } \\
(\mathrm{ML})\end{array}$ & $\begin{array}{c}F_{\text {peak }}^{\text {total }} \\
\left(\mathrm{mJ} / \mathrm{cm}^{2}\right)\end{array}$ & $\begin{array}{c}F_{\text {peak }}^{\mathrm{p}-\text { pol }} \\
\left(\mathrm{mJ} / \mathrm{cm}^{2}\right)\end{array}$ & $\begin{array}{c}F_{\text {peak }}^{\text {s-pol }} \\
\left(\mathrm{mJ} / \mathrm{cm}^{2}\right)\end{array}$ & $\begin{array}{c}\mathrm{HW}_{-} \\
(\mathrm{ps})\end{array}$ & $\begin{array}{c}\text { HW } \\
(\mathrm{ps})\end{array}$ & $n$ \\
\hline \hline 0.24 & 13.1 & 9.3 & 3.8 & 1.1 & 10.4 & $9.3 \pm 0.5$ \\
0.64 & 10.6 & 7.5 & 3.1 & 9.2 & 25.5 & $10.3 \pm 0.4$ \\
0.75 & 7.2 & 5.1 & 2.1 & 6.7 & 17.0 & $6.9 \pm 0.3$ \\
\hline
\end{tabular}

Second, the widths of the correlations are large enough that they do not provide immediate insight into the substrate-adsorbate energy transfer mechanism. Specifically, femtosecond-pulse excitation of the substrate can drive desorption through adsorbate coupling either to electrons or 
phonons. Narrow 2PC widths of $\sim 1$ ps or less indicate unambiguously that electronic coupling is dominant, because only the electrons respond on the subpicosecond timescale. Broader 2PC widths are ambiguous, because they are in principle consistent with weak coupling of the adsorbate to the electrons, or strong coupling to the phonons. The HWHMs in Table I fall in the latter category, with the possible exception of the fast decay at negative delays for the 0.24-ML adlayer. Thus, a more detailed analysis involving numerical modeling is required to elucidate the energy mediation mechanism and extract the coupling strengths.

Third, the nonmonotonic behavior of the HWHMs with coverage show that the dynamics are complex. This suggests that the strong monotonic increase in the desorption probability with increasing coverage above $\sim 0.3 \mathrm{ML}$ shown in Figure 3 cannot be simply explained in terms of a weakening of the molecule-surface bond strength (for the most labile molecules in the adlayer) with coverage. Given that it is known that CO binds $\mathrm{Pd}(111)$ more strongly at three-fold hollow sites than at bridge sites, and is most loosely bound at top sites, one might hypothesize that there are two competing trends: a decrease in the desorption barrier with coverage that enhances the desorption probability and a decrease in the substrate-adsorbate energy coupling strength with coverage that attenuates that enhancement. However, the HWHMs in Table 2PC suggest that the coupling strength, $\eta$, varies with the coverage in a complex way: $\eta(\theta=0.24 \mathrm{ML})>\eta(\theta=0.75$ $\mathrm{ML})>\eta(\theta=0.64 \mathrm{ML})$. This nonmonotonic behavior contradicts a simple competition between barrier height and coupling strength and points again to the need for modeling to provide further insight.

Finally, close inspection of the 2PC data near zero delay reveals that the desorption probability is not a simple decay from a maximum value (Figure 5). Instead, for all coverages we observe a 5-20\% “dip” in the probability at zero delay relative to the maximum desorption signal. 
Such dips have been predicted by 2TM simulations $^{23,} 50$ and observed in transientreflection time-resolved temperature measurements, ${ }^{57}$ but, to the best of our knowledge, have not been seen in any previous 2PC desorption probability measurements. A good model of the energy transfer dynamics should reproduce these dips, with the caveat that the observed amplitude of the dip is possibly reduced due to a "coherent artifact” near zero delay—residual optical interference between the pulses generates hot spots that on average artificially enhance the observed desorption probabilities.

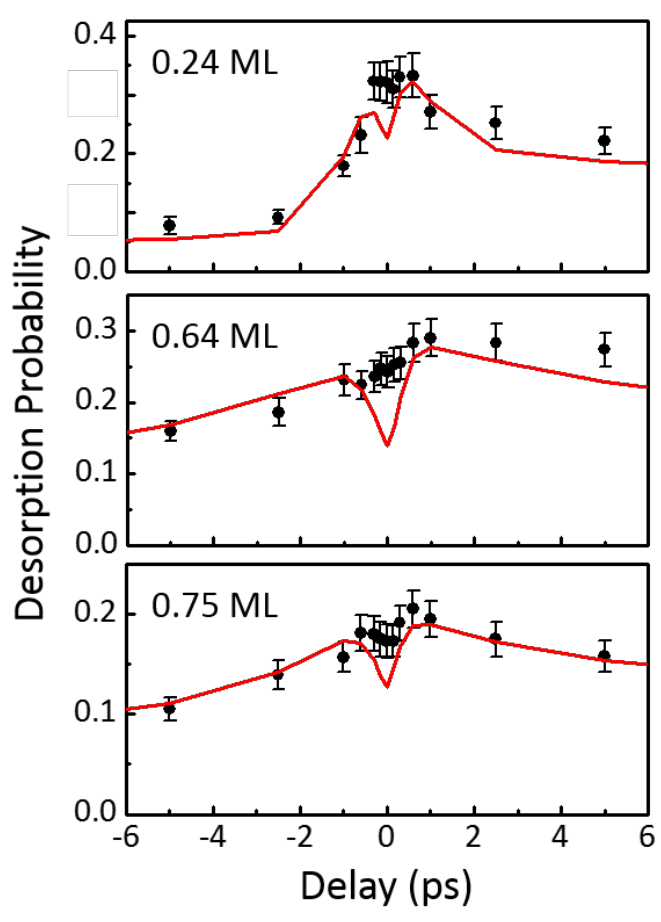

Figure 5. Short delay portions of the 2PC data. These data are the same as shown in Figure 4, but only the short delay portion is shown here to highlight the behavior near zero delay. The solid lines are two-temperature model simulations using constant electronic coupling (Model I) as described in the Numerical Modeling section.

\section{Numerical Modeling Results}

To understand the strong dependence of the desorption probability on coverage-and possibly adsorption site—and to consider the roles played by adsorbate-surface and adsorbateadsorbate interactions in the chemical dynamics, we have employed a set of frictional-coupling models to simulate the temporal evolution of the laser-excited adsorbate-substrate complex. These models predict the fs-PID probability by simulating the temporal evolution of three processes: (i) substrate excitation driven by absorption of the laser pulse(s) and governed by heat transport into the substrate bulk, (ii) vibrational excitation of the adsorbates via frictional coupling, and (iii) desorption by crossing an activation barrier. This approach, which has been referred to as the 
“empirical friction” model, ${ }^{23,49}$ substantially simplifies the physics, but has been used extensively to gain qualitative insight into the energy transfer mechanism and quantify the surface dynamics in terms of phenomenological frictional coupling strengths. ${ }^{19,} 23,31,49,54,58,59$ Direct comparisons with computationally intensive DFT-based molecular dynamics simulations have indicated that the empirical friction model is a practical tool for approximating adsorbate excitation and extracting qualitative trends from experimental data, but that quantitative agreement between the resultant fitting parameter values and the actual physical quantities (e.g., activation energies and friction coefficients) should not be expected..$^{60,61}$

The substrate excitation is described by a two temperature model, ${ }^{62}$ where the metal substrate is modeled as two temperature baths, an electron bath and a lattice bath, characterized by temperatures, $T_{\mathrm{el}}$ and $T_{\mathrm{ph}}$, the electronic and lattice (phonon) temperatures, respectively. The temporal evolution of these temperatures is obtained by numerically solving two coupled differential equations:

$$
\begin{gathered}
C_{\mathrm{el}} \frac{\partial}{\partial t} T_{\mathrm{el}}=\frac{\partial}{\partial z} \kappa \frac{\partial}{\partial z} T_{\mathrm{el}}-g\left(T_{\mathrm{el}}-T_{\mathrm{ph}}\right)+S(t, z) \\
C_{\mathrm{ph}} \frac{\partial}{\partial t} T_{\mathrm{ph}}=g\left(T_{\mathrm{el}}-T_{\mathrm{ph}}\right),
\end{gathered}
$$

where $t$ is time, $z$ is depth into the substrate, $C_{\mathrm{el}}$ and $C_{\mathrm{ph}}$ are the electron and the lattice heat capacities, $\kappa$ is the thermal conductivity, $g$ is the electron-phonon coupling constant, and $S(t, z)$ is the laser heating "source" term. The source term is characterized by the measured laser pulse peak fluence, temporal width, functional form $\left(\operatorname{sech}^{2}\right)$ and wavelength-dependent extinction coefficient. Lateral energy diffusion is ignored because the dimensions of the laser spot $(\sim \mathrm{mm})$ are much larger than the energy diffusion length $(\sim 100 \mathrm{~nm})$ over the duration of the simulated 
events ( 100 ps). Further details and the values used for the Pd substrate parameters can be found in our previous work. ${ }^{50}$

To describe the substrate-adsorbate energy transfer, we adopt the equations derived by Budde et al., ${ }^{63}$ from a master equation formalism, where the adsorbate motion along the reaction (desorption) coordinate is approximated as harmonic. ${ }^{23,49,63}$ This gives the rate of energy transfer between the adsorbate and the two substrate temperature baths as:

$$
\begin{gathered}
\frac{d U_{\mathrm{ads}}}{d t}=\eta_{\mathrm{el}}\left(U_{\mathrm{el}}-U_{\mathrm{ads}}\right)+\eta_{\mathrm{ph}}\left(U_{\mathrm{ph}}-U_{\mathrm{ads}}\right) \\
U_{q}=\frac{h v_{\mathrm{rc}}}{\exp \left(h v_{\mathrm{rc}} / k_{\mathrm{B}} T_{\mathrm{q}}\right)-1},
\end{gathered}
$$

where $\eta_{\mathrm{el}}\left(\eta_{\mathrm{ph}}\right)$ is the strength of the frictional coupling between the adsorbate and the electron (phonon) bath, $v_{\mathrm{rc}}$ is the vibrational frequency of the adsorbates along the reaction (desorption) coordinate, $U_{\text {ads }}$ is the average vibrational energy in that coordinate, characterized by adsorbate temperature $T_{\mathrm{ads}}$, and $U_{\mathrm{el}}\left(U_{\mathrm{ph}}\right)$ is what the average vibrational energy would be if the adsorbates equilibrated at $T_{\mathrm{el}}\left(T_{\mathrm{ph}}\right)$. Thus Equation 5 permits simulation of the time dependence of the adsorbate temperature from the temporal profile of the two substrate temperature baths. The coupling coefficients can be understood as the inverse time constants characteristic of the energy transfer rate $\left(\eta_{\mathrm{el}}=1 / \tau_{\mathrm{el}}, \eta_{\mathrm{ph}}=1 / \tau_{\mathrm{ph}}\right)$. In our simulations, $v_{\mathrm{rc}}$ is taken to be $10 \mathrm{THz}$ which is equal to the frequency of the CO-Pd stretch calculated with DFT by Gajdoš et al. ${ }^{48}$ That calculation showed that the frequencies varied with adsorption site: 9.4, 9.5, 10.4 and $12.3 \mathrm{THz}$ at the fcc $3 \mathrm{fh}$, hcp 3fh, bridge and top sites, respectively. Because a 25\% variation in $v_{\mathrm{rc}}$ had a negligible ( 5 \%) effect on the simulated desorption yields, we have chosen to keep the model simple by using a single, site-independent value.

The desorption rate is calculated using the Polanyi-Wigner (PW) equation: 


$$
R_{\mathrm{d}}=-\frac{d \theta}{d t}=\theta v_{\mathrm{PW}} \exp \left(-E_{\mathrm{a}} / k_{\mathrm{B}} T_{\mathrm{ads}}\right)
$$

where $\theta$ is the coverage, $v_{\mathrm{PW}}$ is the prefactor, $E_{\mathrm{a}}$ is desorption activation energy and $k_{\mathrm{B}}$ is the Boltzmann constant. We used the “inversion-optimization" method ${ }^{64}$ to find $v_{\mathrm{PW}}=158 \mathrm{THz}$ (assumed independent of coverage), and $E_{\mathrm{a}}=1.38,0.78$ and $0.50 \mathrm{eV}$, for $0.24,0.64$ and $0.75 \mathrm{ML}$, respectively, from a series of $\mathrm{CO} / \mathrm{Pd}(111) \mathrm{TPD}$ measurements, where the initial coverage was varied. The temporal evolution of $R_{\mathrm{d}}$ is calculated using these thermal desorption parameters along with the simulated temporal evolution of $T_{\mathrm{ads}}$, and the fs-PID yield is simulated by integrating $R_{\mathrm{d}}$ until the end of the simulation, which is a few hundred picoseconds beyond arrival of the second pulse, ensuring that the desorption has ended.

To qualitatively understand the energy transfer physics, we tested four substrate-adsorbate coupling models: Model I employed constant electronic coupling only; Model II, temperaturedependent electronic coupling only; Model III, electronic plus phononic coupling; and Model IV, phononic coupling only. The fit of the models was judged first on their ability to reproduce the 2PC results for the selected coverages, $0.24,0.64$, and $0.75 \mathrm{ML}$, and second on the predicted value of the power-law exponent.

With the exception of the coupling strengths $\left(\eta_{\mathrm{el}}\right.$ and $\left.\eta_{\mathrm{ph}}\right)$, all simulation parameters were fixed as described above. However, we found it necessary to make two adjustments to the parameters used for the 0.75-ML adlayer. First, because the desorption probability at this coverage asymptotically approaches $\sim 0.3$ and not unity with increasing fluence, we concluded that only onethird of the adsorbates were being desorbed. This is not unreasonable, given that two-thirds of the molecules in the $(2 \times 2)$ adlayer are tightly-bound in 3fh sites (Figure 1) and that the desorption probability from these sites is $~ 100$ times less than what is observed once the top sites are populated 
(Figure 3). The experimental probabilities in all figures in this report do not reflect this adjustment, because we have elected to show these as uncorrected probabilities (i.e., the ratio of the measured desorption yield to the measured total coverage) without imposing this interpretation. The simulated probabilities reported for the 0.75-ML case, however, do reflect this interpretation, as follows. In the 0.75-ML simulations the coverage in the calculations was set to 0.25 ML (i.e., only top-site $\mathrm{CO}$ is allowed to desorb), and the simulated probabilities were divided by three for comparison with the experimental measurements. Second, the desorption activation energy, $E_{\mathrm{a}}$, was increased from the experimentally estimated value of 0.50 to $0.54 \mathrm{eV}$. This was necessary because simulated 2PCs were too broad for all values of $\tau_{\text {el }}$ that gave reasonable fs-PID probabilities. We found that a small increase in $E_{\mathrm{a}}$ made it possible to reproduce the observed 2PC width. The uncertainty in the estimated $E_{\mathrm{a}}$ is easily large enough to accommodate this adjustment, and we made the smallest increase possible such that the 2PC width could be approximated in the simulations.

For Models I, II and III, the coupling strengths were optimized by minimizing the goodness of fit function,

$$
\chi_{2 \mathrm{PC}}^{2}=\frac{1}{f} \sum\left(\frac{P_{\exp }-P_{\mathrm{sim}}}{\sigma_{\exp }}\right)^{2},
$$

where and $P_{\exp }$ and $P_{\text {sim }}$ are the experimentally determined and simulated values of the 2PC desorption probabilities, $\sigma_{\text {exp }}$ are the estimated relative uncertainties in the probabilities based on standard deviation of the mean for the data at each delay, and the sum is over all measured points. To compare the Models, Equation 7 accounts for the number of degrees of freedom, $f=m-p$, where $m$ is the number of data points ( $m=23$ for all data sets) and $p$ is the number of parameters ( $p=1,3,2$ and 1, for Models I, II, III and IV, respectively). Model I, II and III simulation results 
are summarized in Tables II and III, and a tabulation of the $\chi_{2 \mathrm{PC}}^{2}$ values is provided in the supplemental material. ${ }^{53}$ As discussed below, Model IV cannot account for the observed desorption probabilities or 2PC timescales.

Model I: Constant Electronic Coupling. Model I reproduces the experimental observations reasonably well, despite its simplicity. We find fairly good agreement between the simulated and observed 2PC measurements (Figures 4 and 5). The best fits were found by varying $\tau_{\mathrm{el}}$ to find the minimum $\chi_{2 \mathrm{PC}}^{2}$ at each coverage. The derived coupling times are listed in Table II. As would be anticipated from the widths of the 2PCs (Table I), the coupling times are ordered: $\tau_{\mathrm{el}}(\theta=0.24 \mathrm{ML})<\tau_{\mathrm{el}}(\theta=0.75 \mathrm{ML})<\tau_{\mathrm{el}}(\theta=0.64 \mathrm{ML})$. This same qualitative trend is found in Models II and III, discussed below. The subpicosecond value for $\tau_{\mathrm{el}}$ at $\theta=0.24 \mathrm{ML}$ is a clear hallmark of electron-mediated energy transfer. ${ }^{20,21}$ Furthermore, all of the $\tau_{\text {el }}$ values are shorter than the 3.6 -ps substrate electron-phonon coupling time. We interpret this as indicating that the CO is well coupled to the Pd electronic degrees of freedom and the fs-PID is driven by electronmediated energy transfer at all coverages. This conclusion is borne out by the Model III and IV simulations discussed below, where the possible role of phonon-mediated energy transfer is considered.

The simplicity of Model I is attractive, and the resulting characteristic coupling times are instructive. However, though Model I reproduces the 2PC data reasonably well, it does not reproduce two features of the full data set: Model I overestimates the magnitude of the zero-delay dips by a factor of $\sim 2-3$, and it underestimates the power-law exponent by $\sim 1-4$. These discrepancies motivate us to consider more sophisticated models, in particular those that incorporate temperature-dependent friction or phononic coupling. 


\section{Table II}

The desorption activation energies used in all simulations, the best-fit coupling times obtained in Model I, II and III simulations, and the power-law exponents for the fluence dependences. For Model II, coupling times are taken at the peak electronic temperature. For Model III, each row gives values from the best-fits (top) and the estimated lower-limit $\tau_{\mathrm{ph}}$ times for 0.24 and $0.75-\mathrm{ML}$ and $\tau_{\mathrm{ph}}=0.5 \mathrm{ps}$ at $0.64 \mathrm{ML}$ (bottom), as explained in the text.

\begin{tabular}{|c|c|c|c|c|c|c|c|c|}
\hline \multirow[b]{2}{*}{$\begin{array}{l}\text { Coverage } \\
\text { (ML) }\end{array}$} & \multirow[b]{2}{*}{$\begin{array}{c}E_{\mathrm{a}} \\
(\mathrm{eV})\end{array}$} & \multicolumn{2}{|c|}{$\begin{array}{c}\text { Model I } \\
\text { constant } \eta_{\mathrm{el}}\end{array}$} & \multicolumn{2}{|c|}{$\begin{array}{c}\text { Model II } \\
T_{\mathrm{el}} \text {-dependent } \eta_{\mathrm{el}}\end{array}$} & \multicolumn{3}{|c|}{$\begin{array}{l}\text { Model III } \\
\eta_{\mathrm{el}}+\eta_{\mathrm{ph}}\end{array}$} \\
\hline & & $\begin{array}{c}\tau_{\mathrm{el}} \\
(\mathrm{ps})\end{array}$ & $n$ & $\begin{array}{c}\tau_{\mathrm{el}} \text { at } T_{\mathrm{el}}^{\text {peak }} \\
\text { (ps) }\end{array}$ & $n$ & $\begin{array}{c}\tau_{\mathrm{el}} \\
(\mathrm{ps})\end{array}$ & $\begin{array}{l}\tau_{\mathrm{ph}} \\
(\mathrm{ps})\end{array}$ & $n$ \\
\hline 0.24 & 1.38 & 0.81 & 5.5 & 0.82 & 6.4 & $\begin{array}{l}0.81 \\
0.75\end{array}$ & $\begin{array}{l}\infty \\
10\end{array}$ & $\begin{array}{l}5.5 \\
5.3\end{array}$ \\
\hline 0.64 & 0.78 & 3.32 & 8.1 & 2.59 & 9.9 & $\begin{array}{l}2.88 \\
2.05\end{array}$ & $\begin{array}{l}1.8 \\
0.5\end{array}$ & $\begin{array}{l}8.1 \\
8.2\end{array}$ \\
\hline 0.75 & 0.54 & 2.48 & 5.7 & 2.02 & 6.2 & $\begin{array}{l}2.48 \\
2.45\end{array}$ & $\begin{array}{c}\infty \\
6.2\end{array}$ & $\begin{array}{l}5.7 \\
5.6\end{array}$ \\
\hline
\end{tabular}

Model II: Temperature-Dependent Electronic Coupling. First, we consider including a $T_{\mathrm{el}}$-dependent electronic friction, ${ }^{19,50,54,65,66}$ with the understanding that it may provide a way to capture energy transfer via both frictional coupling and desorption induced by multiple electronic transitions (DIMET) ${ }^{67}$ where the importance of a DIMET contribution is understood to depend on the overlap of the adsorbate orbitals and the substrate hot-carrier distribution, and therefore on the electronic temperature. ${ }^{30,67}$ Alternatively, the $T_{\mathrm{el}}$-dependent friction may serve as a proxy for heat transfer via mode coupling, ${ }^{59,68,69}$ a process that is beyond the scope of our measurements and simple simulations. In any case, we parameterize the electronic coupling as

$$
\eta_{\mathrm{el}}\left(T_{\mathrm{el}}\right)=\eta_{0}+\eta_{\alpha} T_{\mathrm{el}}^{\alpha}
$$


where $\eta_{0}, \eta_{\alpha}$, and $\alpha$ are adjusted to model the 2PC data. This model, Model II, outperforms Model I in all respects: At all coverages it gives a smaller $\chi_{2 \mathrm{PC}}^{2}$, a value for the power-law exponent $n$ in better agreement with experiment (Table II and Figure 6), and an improved modeling of the zero-delay dip (Figure 7).

The parameters for the temperaturedependent electronic friction are given in Table III. We list the parameters that gave the best-fit with $\alpha$ fixed at 1.75 , the value that best modelled our previously-published measurements. ${ }^{54}$ We also show results for $\alpha$ values that gave slightly

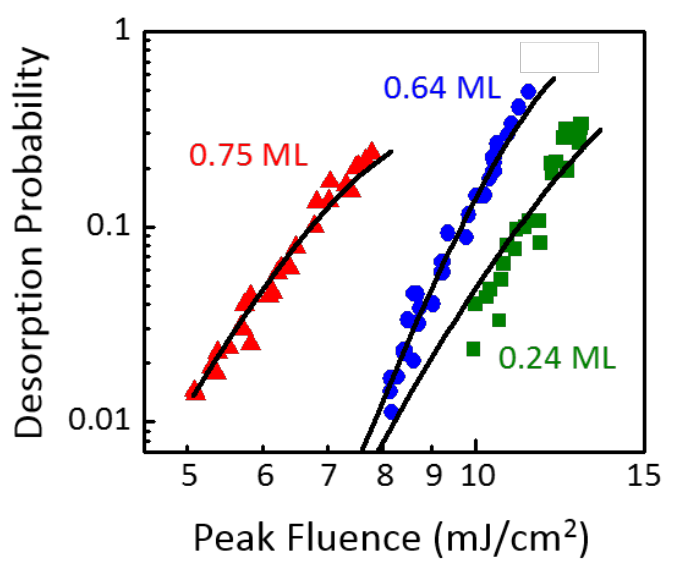

Figure 6. Temperature-dependent electronic friction simulations (Model II, $\alpha=1.75$ ) of the fluence dependence of the $\mathrm{CO}$ fs-PID probabilities (lines). The experimentally measured values at 0.24 (squares), 0.64 (circles), 0.75 ML (triangles) are the same as those in Figure 2.
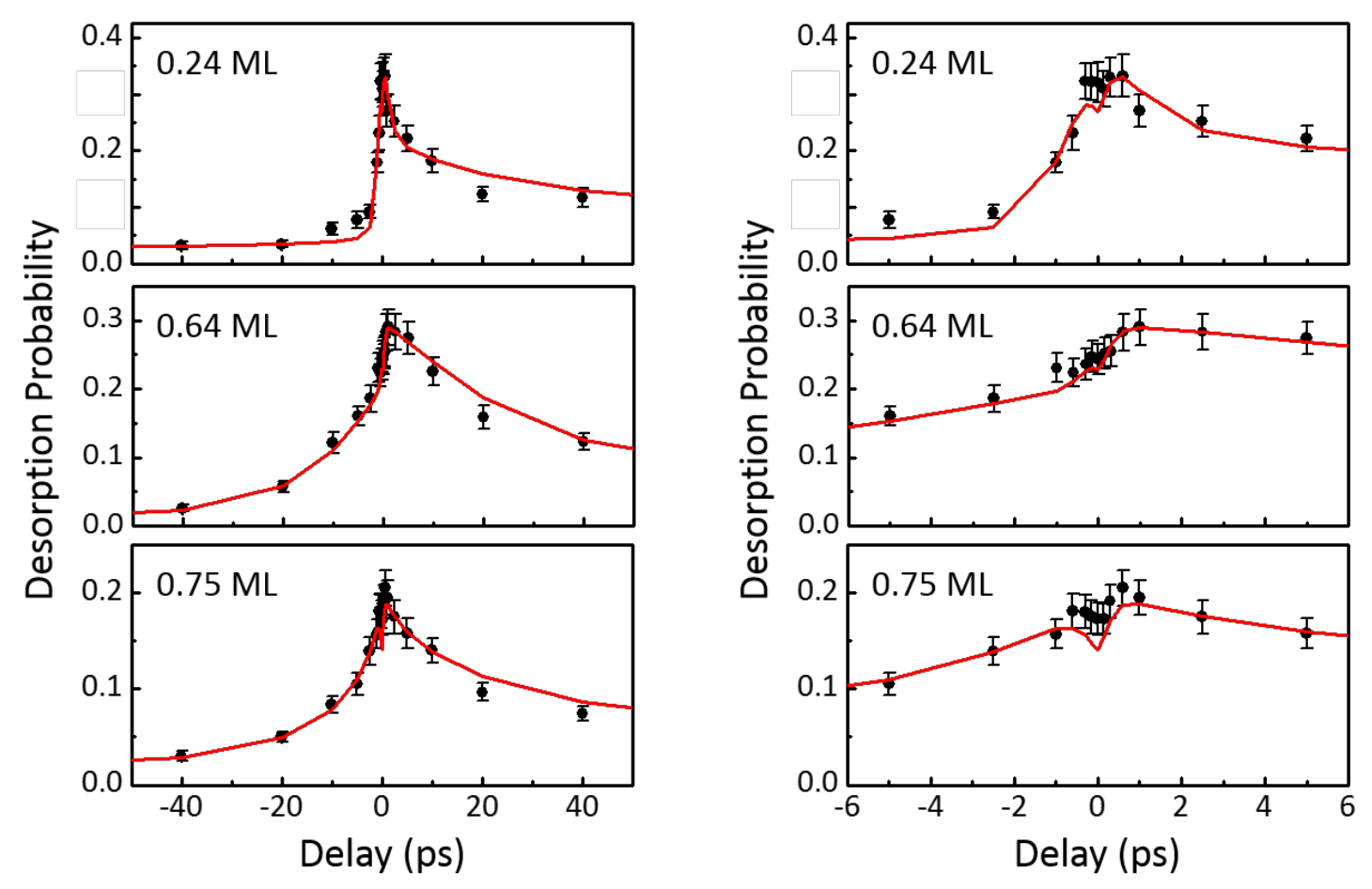

Figure 7. Empirical friction simulations (solid lines) using temperature-dependent electronic coupling (Model II, $\alpha=1.75$ ) overlaid on the 2PC data as in Figures 4 and 5. 
improved fits at 0.24 and $0.75 \mathrm{ML}(\alpha=1.75$ gave the best fit at $0.64 \mathrm{ML})$. Comparison of the best-fit $n$ values, peak adsorbate temperatures, $T_{\mathrm{ads}}^{\text {peak }}$, and coupling times at peak excitation, $\tau_{\text {el }}$ at $T_{\mathrm{el}}^{\text {peak }}$, illustrate that the choice of alpha within the range of 1 to 2.5 is not critical. As judged by the values of $\tau_{\mathrm{el}}$ at $T_{\mathrm{el}}^{\mathrm{peak}}$, the trend in coupling times found with Model I is reproduced by Model II: $\tau_{\mathrm{el}}(\theta=0.24 \mathrm{ML})<\tau_{\mathrm{el}}(\theta=0.75 \mathrm{ML})<\tau_{\mathrm{el}}(\theta=0.64 \mathrm{ML})$.

\section{Table III}

Friction parameters that give the best Model II fit to the 2PC measurements. The electronic coupling times at the peak electronic temperature and the adsorbate temperature at the desorption rate peak derived from the fits, assuming an absorbed fluence of $7.76 \mathrm{~mJ} / \mathrm{cm}^{2}$, are given. For the 0.24 - and 0.75-ML adlayers, bestfit values for $\alpha$ fixed at 1.75 are also shown.

\begin{tabular}{ccccccc}
\hline $\begin{array}{c}\text { Coverage } \\
(\mathrm{ML})\end{array}$ & $\alpha$ & $\begin{array}{c}\eta_{0} \\
\left(\mathrm{ps}^{-1}\right)\end{array}$ & $\begin{array}{c}\eta_{\alpha} \\
\left(\mathrm{s}^{-1} \mathrm{~K}^{-\alpha}\right)\end{array}$ & $\begin{array}{c}\tau_{\mathrm{el}} \text { at } T_{\mathrm{el}}^{\text {peak }} \\
(\mathrm{ps})\end{array}$ & $n$ & $\begin{array}{c}T_{\mathrm{ads}}^{\text {peak }} \\
(\mathrm{K})\end{array}$ \\
\hline \hline \multirow{2}{*}{0.24} & 1.75 & 0.86 & $1.7 \times 10^{5}$ & 0.82 & 6.4 & 1652 \\
& 1.00 & 0.55 & $1.6 \times 10^{8}$ & 0.82 & 6.4 & 1629 \\
0.64 & 1.75 & 0.11 & $1.3 \times 10^{5}$ & 2.59 & 9.9 & 762 \\
& & & & & & \\
& & & & & & \\
0.75 & 1.75 & 0.30 & $9.2 \times 10^{4}$ & 2.02 & 6.2 & 983 \\
& 2.50 & 0.32 & $1.9 \times 10^{2}$ & 1.91 & 6.3 & 994 \\
\hline
\end{tabular}

Model III: Electronic plus Phononic Coupling. Because ultrafast photoexcitation of the Pd imparts energy to both the electronic and lattice degrees of freedom (Equation 4), and both baths are, in principle, coupled to the adsorbates (Equation 5), we used Model III to investigate the possible involvement of phonon-mediated energy transfer in the observed dynamics. Within the context of the empirical friction model, we ask: Is phonon-mediated energy transfer significant 
on the timescales of the fs-PID process for the CO/Pd(111) system? To answer this question, we systematically varied $\tau_{\mathrm{ph}}$ over a broad range of values, from the subpicosecond out to the millisecond regime. We used the same $\chi_{2 \mathrm{PC}}^{2}$ (Equation 7) to search for the set of coupling times, $\tau_{\mathrm{el}}$ and $\tau_{\mathrm{ph}}$ (Equation 5), that best fit the data. We found that best fits for Model III for 0.24 and $0.75 \mathrm{ML}$ were obtained with $\tau_{\mathrm{ph}}=\infty \mathrm{ps}$, and for $0.64 \mathrm{ML}$ with $\tau_{\mathrm{ph}}=1.8 \mathrm{ps}$. However, we also found that for 0.24 and $0.75 \mathrm{ML}$ finite vales of $\tau_{\mathrm{ph}}$ gave only slightly larger $\chi_{2 \mathrm{PC}}^{2}$ values. Specifically, $\tau_{\mathrm{ph}}=10$ and $6.2 \mathrm{ps}$ for 0.24 and $0.75 \mathrm{ML}$, respectively, increased $\chi_{2 \mathrm{PC}}^{2}$ only $10 \%$, and gave 2PC simulations that, to the eye, were very similar to those in Model I (i.e., $\tau_{\mathrm{ph}}=\infty$ ). We report these $\tau_{\mathrm{ph}}$ values in Table II, then, as estimated lower limits on the phononic coupling strengths for the $(\sqrt{3} \times \sqrt{3}) R 30^{\circ}$ and $(2 \times 2)$ adlayers. Furthermore, for the $0.64-\mathrm{ML}$ case, we find that the $\chi_{2 \mathrm{PC}}^{2}$ minimum is very shallow: any value of $\tau_{\mathrm{ph}}$ between 0.5 and $\infty$ ps gives $\chi_{2 \mathrm{PC}}^{2}$ values that are not more than 5\% larger than that found at the minimum. We conclude, then, that Model III alone does not give any clear information regarding the role of phononic coupling to the $c(\sqrt{3} \times 5)$ adlayer. Because the $2 \mathrm{PC}$ timescale is so long, this is not unexpected. With this caveat, for the $0.64-\mathrm{ML}$ adlayer we report in Table II the parameters that gave the minimum $\chi_{2 \mathrm{PC}}^{2}$ value, along with those for $\tau_{\mathrm{ph}}=0.5 \mathrm{ps}$.

To summarize the Model III results, then, comparison with Model I shows that inclusion of phonon-mediated energy transfer does not provide an improved description of the 2PC data. It also makes no improvement in the power-law exponent. However, it is also clear that we cannot rule out weak adsorbate-phonon coupling. This does not mean that the phonons are not coupled to the adsorbate, however. One reasonable scenario is that the adsorbate-phonon coupling timescale is simply too long to be unambiguously identified in the 2PC and fluence dependence 
data. Thus coupling to the electrons drives the desorption, while coupling to the phonons moderates the degree of adsorbate excitation. Based on the 10-ps lower limit estimated for the 0.24-ML case, and a theoretical prediction that the phononic coupling strength scales with coverage for periodic adlayers, ${ }^{70}$ we can estimate coverage-dependent lower limits for the phononic coupling times. For the 0.64 and 0.75-ML adlayers we would predict 3.75 and $3.2 \mathrm{ps,}$ respectively. In such a case, Model III gives electronic coupling times of 0.75, 3.11 and 2.05 ps for the $0.24,0.64$ and $0.75-\mathrm{ML}$ adlayers, respectively. These times are somewhat shorter than those predicted by Model I. Nevertheless the qualitative conclusions based on Model I remain accurate: (i) electronic coupling drives the desorption and (ii) the electronic coupling times increase in the order: $\tau_{\mathrm{el}}(\theta=0.24 \mathrm{ML})<\tau_{\mathrm{el}}(\theta=0.75 \mathrm{ML})<\tau_{\mathrm{el}}(\theta=0.64 \mathrm{ML})$. The physics behind trend will be considered in the discussion section.

Model IV: Phononic Coupling. Finally, we consider whether coupling to phonons alone (Model IV) can reproduce the observed fs-PID probabilities and 2PC results. Simulations using Model IV with $\tau_{\mathrm{ph}}=1$ ps show that despite reaching peak temperatures ranging from 500 to $1500 \mathrm{~K}$ when excited at peak fluences in the 7 to $13 \mathrm{~mJ} / \mathrm{cm}^{2}$ range, phonons alone cannot account for the high fs-PID probabilities, nor the comparatively narrow widths of the 2PCs (Figure 8). The failure of Model IV is most pronounced at 0.24 ML, where the simulated fs-PID probability is two orders of magnitude lower and the simulated 2PC width two times larger than the measured values. The discrepancy in yield is a result of the fact that the phonon bath is not hot enough to heat the CO sufficiently to overcome the high activation barrier for desorption from $3 \mathrm{fh}$ sites at this coverage. At the higher coverages, where the activation barrier is smaller, the failure of Model IV to reproduce the fs-PID probabilities is less dramatic. However, the model still overestimates the 
2PC widths, particularly at negative delays where the measurement is most sensitive to short coupling times.

Direct experimental access to adsorbatephonon coupling times remains a challenge. ${ }^{34,71-}$ ${ }^{73}$ Available data from pump-probe laser experiments that use the high-frequency $\mathrm{C}-\mathrm{O}$ stretch as an indirect probe, ${ }^{4,34,74}$ and inelastic Heatom scattering, ${ }^{75}$ as well as theoretical simulations ${ }^{6}, 70,76$ generally indicate phonon damping lifetimes in the $\sim 1-10$ ps range for the most investigated system, $\mathrm{CO}$ on $\mathrm{Cu}$ surfaces. Nevertheless, we also considered the possibility

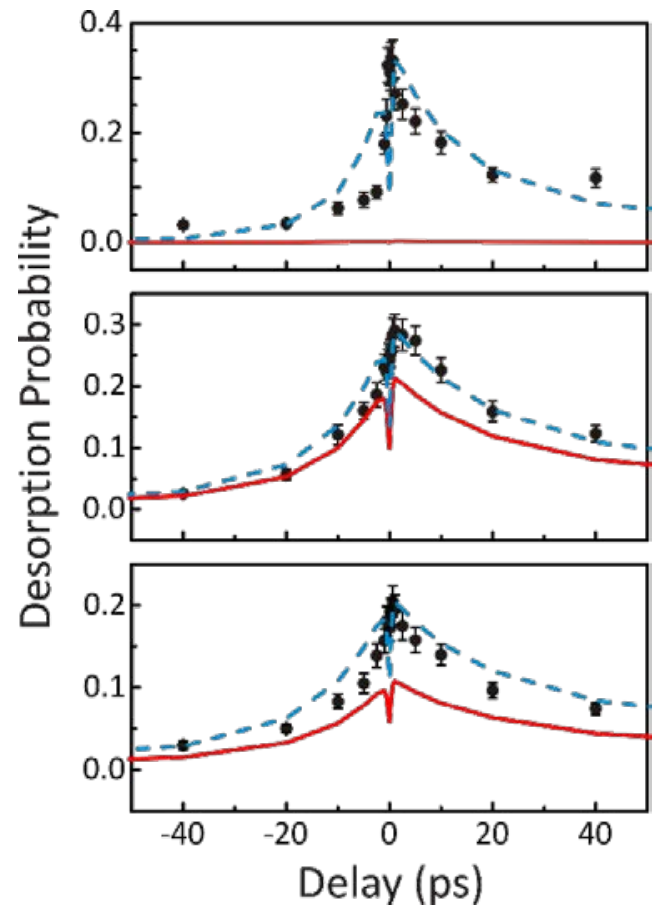

Figure 8. Comparison of the phonon coupling model 2PC simulations with the experimental measurements (circles) at coverages of 0.24, 0.64, and $0.75 \mathrm{ML}$ (top to bottom): as calculated (solid line) and normalized to the peak experimental probabilities (dashed line).

that the phononic coupling times may be subpicosecond, such as those used recently to model $\mathrm{CO} / \mathrm{Ru}(0001) .{ }^{52}$ Based on measurements and calculations of the adsorbate-metal vibrational modes (frustrated translation, frustrated rotation and molecule-metal stretch) for a range of similar systems, ${ }^{48,72,77}$ the frustrated translation may be in resonance with the substrate phonon bath, and if so, would be expected to result in subpicosecond adsorbate-phonon coupling times. To check whether very short phononic coupling times could explain our observations, we ran 2TM simulations with $\tau_{\mathrm{ph}}$ as short as $0.1 \mathrm{ps}$. We found that peak yields were increased by at most $15 \%$, and the 2PC widths remained essentially unchanged.

Thus, for the 0.24-ML adlayer, subpicosecond coupling to the phonon bath does little to improve the agreement, and we conclude that phonon-driven energy transfer cannot explain the 
observed low coverage dynamics. The situation at the higher coverages, however, is not as unambiguous. Given that the empirical friction model is a simplification of the physics, and that the parameters used to describe the substrate and the adsorbate are not known to high precision, we must leave room for the possibility that at high coverages the energy transfer is phonon mediated. For example, increases of the order of $50 \%$ in the electron-phonon coupling time or the PW pre-exponential would bring the Model IV simulated fs-PID probabilities close to agreement with the observed values. However, we have not found a set of reasonable parameters that is able to reproduce the relatively short timescale of the dynamics at $0.75 \mathrm{ML}$, nor the nonmonotonic behavior of the 2PC width. Taking into consideration that Models I, II and III all indicate that coupling to a higher-temperature, faster-responding energy bath than the phonon bath is needed to drive the desorption, we are constrained to discard Model IV.

To summarize the modeling results, the observed dynamics are most simply explained by electron-mediated energy transfer. If phonons play a role, they act to moderate the level of adsorbate excitation. We also find that the model that best fits the 2PC profiles and fluencedependence data is one that includes an electronic friction that depends on the electronic temperature. In the discussion that follows we consider how a site-dependent electronic coupling strength provides a satisfying explanation for the experimental and numerical modeling results. We also consider the limitations of the empirical friction model and opportunities for further investigation.

\section{Discussion}

The coverage dependences of the fs-PID probability, power-law exponent and 2PC timescales lead to two salient observations: (i) the substrate-adsorbate energy coupling depends 
on coverage and (ii) that dependence is nonmonotonic. In the following we argue that the coupling coverage dependence can be assigned primarily to an adsorption-site dependence, viz., where CO is most strongly coupled to the Pd at hollow sites, less so at bridge sites, and most weakly at top sites. We explain how this is consistent with the dramatic increase in yield with increasing coverage from 0.24 to $0.75 \mathrm{ML}$ and propose that our findings suggest interadsorbate energy transfer from the strongly-coupled 3fh-site CO to the weakly-coupled top-site CO as a natural explanation for the nonmonotonicity of the coupling times. This interadsorbate energy transfer results in an indirect coupling of the substrate electronic excitation to the top-site $\mathrm{CO}$ at a rate faster than would be expected based on the electronic structure of the three binding sites alone. We also put our findings in the context of similar measurements on other systems, and speculate regarding the increase in fs-PID probability with decreasing coverage below 0.3 ML.

The evidence for coverage-dependent electron-mediated coupling is straightforward: (i) the measured 2PC width depends on coverage, and (ii) that variation is well reproduced by electronic-coupling empirical friction models that reproduce the observed fs-PID probabilities (Figures 4, 5 and 7) with coverage-dependent frictions. Thus far, however, we have not discussed the fact that the coverage dependence of the fs-PID probability alone implies a coverage-dependent coupling. This is shown in Figure 9, where we show fs-PID probabilities calculated using Model I, constant electronic coupling, for a series

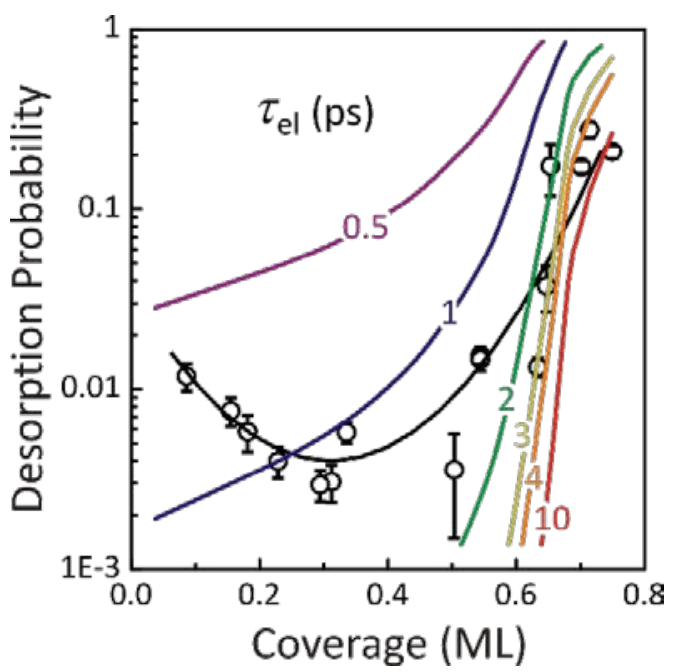

Figure 9. The coverage dependence of the fs-PID probability calculated with Model I using the coverage dependent activation energies derived from TPD and selected constant electronic friction values, $\eta_{\mathrm{el}}=1 / \tau_{\mathrm{el}}$, as marked (solid lines), overlaid on the experimental data (circles) and line to guide the eye. 
of fixed coupling times. In this calculation, only the desorption activation energy, $E_{\mathrm{a}}$, is varied with coverage according to the values determined from TPD measurements. Figure 9 is instructive because it graphically shows that no single coupling time explains the observed trend. Instead, from the measured fs-PID probability and $E_{\mathrm{a}}$ values alone, we can deduce coverage-dependent coupling times of $\sim 1$ and $\sim 2-3$ ps for 0.24 and 0.64 ML, respectively, in agreement with the values derived from the 2PC simulations (Table II). For $0.75 \mathrm{ML}$ we would arrive at a value of $~ 5-10 \mathrm{ps}$ if we assume that all $\mathrm{CO}$ molecules in the adlayer have an equal desorption probability, regardless of adsorption site. This estimate is obviously at odds with the observed 2PC width. However, if we instead assume that only the top-site population of the $0.75 \mathrm{ML}$ adlayer is labile, Figure 9 gives an estimate of $\sim 2-3$ ps for the coupling time. This value matches that derived from the numerical simulations.

Figure 9 makes clear a concept not immediately obvious from the detailed numerical modeling. Namely, we see that if the coupling were not decreasing with coverage, the probability would rise even more steeply than the observed factor of $\sim 100$, illustrating the partially compensating trends: as $E_{\mathrm{a}}$ decreases with coverage, so must $\eta_{\mathrm{el}}$, to moderate the increase in fs-PID probability that would otherwise be much steeper. Thus the magnitude of the difference between the fs-PID probabilities at low coverage and near-saturation coverage indicates that the coupling generally decreases with coverage.

This general trend is intuitively satisfying: the more loosely-bound the adsorbate is to the surface, the more weakly coupled it is to the substrate electrons. However, all site-dependent trends related to the electronic structure of the adsorbate-substrate complex suggest that there should be a monotonic change in electron-mediated energy transfer rates. That the trend is nonmonotonic, 
thus, leads us to confront the question: Why does the coupling to top-site CO appear to be stronger than that to bridge-site $\mathrm{CO}$ ?

Two explanations for the observed behavior merit discussion. First, it is conceivable that the bridge site has anomalously weak electronic coupling. Hypothetically, this could be attributed to a lower electron density over the bridge site, such as recently predicted for bridge sites on $\mathrm{Ag},{ }^{78}$ or to differences in symmetry over the bridge site as compared to the $3 \mathrm{fh}$ and top site. ${ }^{79}$ It is conceivable that such factors could lower the electronic friction at the bridge site compared to both the $3 \mathrm{fh}$ and top sites. While we cannot rule such a scenario out, we think it unlikely given the above-mentioned monotonic trends in the site dependence of the electronic and geometric structure.

Alternatively, it seems very likely that interadsorbate energy transfer enhances the energy transfer rate from the substrate to the top-site $\mathrm{CO}$. The molecular packing density at $0.75 \mathrm{ML}$ is quite high: the CO density in the $(2 \times 2)$ adlayer is 1.8 times that of the $(001)$ plane of solid $\mathrm{CO}^{80}$ and the nearest-neighbor CO separation (3.175 $\AA$ ) is only 93\% the van der Waals diameter of carbon. ${ }^{81}$ Thus the $3 \mathrm{fh} \mathrm{CO}$ are in close contact with the copopulation of top-site CO. Furthermore, the 3 fh CO should be nearly as strongly coupled to the substrate in the $(2 \times 2)$ adlayer as they are in the $(\sqrt{3} \times \sqrt{3}) R 30^{\circ}$, given that the C-surface distance for 3fh-CO is calculated to be only slightly larger at saturation compared to the low coverage: 1.366 and $1.296 \AA$, respectively. ${ }^{45}$ The tightly-bound 3fh CO would not be expected to desorb at the relatively low laser fluences used to excite the $(2 \times 2)$ adlayer, however, their vibrational modes would still be efficiently excited. This excitation should be efficiently transferred to the top-site molecules as a result of repulsive interactions ${ }^{77,82}$ between neighbors or dynamic changes in the potential energy surface, ${ }^{83}$ or both effects, in the tightly-packed adlayer, resulting in desorption of the more labile top-site CO. 
A similar mechanism was proposed to explain the promotion of recombinative $D_{2}$ desorption by interadsorbate energy transfer from rapidly excited $\mathrm{H}$ atoms to slower-responding $\mathrm{D}$ atoms in fs-PID of mixed $\mathrm{H}+\mathrm{D}$ adlayers on $\mathrm{Ru}(001) .{ }^{58}$ That the lighter $\mathrm{H}$ atoms were more rapidly excited than the $\mathrm{D}$ atoms was confirmed by $2 \mathrm{PC}$ measurements of pure $\mathrm{H}_{2}$ and $\mathrm{D}_{2}$ adlayers. Interadsorbate coupling was invoked to account for observed deviations from expected yields in the mixed adlayers.

To quantify the interadsorbate energy transfer in the 0.75-ML CO adlayer on Pd, we modified Model I by describing the energy flow with two expressions:

$$
\begin{aligned}
& \frac{d U_{\mathrm{ads} 1}}{d t}=\eta_{\mathrm{el} 1}\left(U_{\mathrm{el}}-U_{\mathrm{ads} 1}\right)+\eta_{\text {inter }}\left(U_{\mathrm{ads} 2}-U_{\mathrm{ads} 1}\right) \\
& \frac{d U_{\mathrm{ads} 2}}{d t}=\eta_{\mathrm{el} 2}\left(U_{\mathrm{el}}-U_{\mathrm{ads} 2}\right)+2 \eta_{\mathrm{inter}}\left(U_{\mathrm{ads} 1}-U_{\mathrm{ads} 2}\right)
\end{aligned}
$$

where indices 1 and 2 correspond to 3fh- and top-site CO, respectively, and $\eta_{\text {inter }}$ is an interadsorbate coupling coefficient. These equations replace Equation 5a, and the thusly-modified model simulates the desorption probability of the two types of CO. The factor of two in the last term of Equation 9b reflects the 2:1 ratio of the 3fh- to top-site CO in the $(2 \times 2)$ adlayer. Because the number of free parameters is large, we employ here only Model I-type simulations, ignoring phononic coupling and the possibility of temperature-dependent electronic coupling. The value of $\eta_{e l 1}$ was fixed at $(0.81 \mathrm{ps})^{-1}$, as determined with Model I for 3fh-CO at 0.24 ML (Table II).

This interadsorbate version of Model I simulates the data well, generating 2PCs that are nearly indistinguishable from that generated with a single coupling time (Figures 4 and 5, lower panels). However, the model is underdetermined: there is an infinite set of $\eta_{\mathrm{el} 2}$ and $\eta_{\text {inter }}$ values that yield solutions that model the data well. Specifically, good fits are found for the set of $\eta_{\mathrm{el} 2}$ and $\eta_{\text {inter }}$ that obey: 


$$
\frac{1}{\tau_{\mathrm{obs}}}=\frac{3}{2}\left(\frac{1}{\tau_{\mathrm{inter}}+\tau_{\mathrm{el} 1}}\right)-\frac{1}{\tau_{\mathrm{el} 2}}
$$

where $\tau_{\mathrm{obs}}$ is the observed overall 2PC coupling time $(2.48 \mathrm{ps}), \tau_{\text {inter }}=1 / \eta_{\text {inter }}$, and $\tau_{\text {el } i}=$ $1 / \eta_{\mathrm{el} i}$. This result is not entirely satisfying, because we would have wished to extract a single value for $\tau_{\text {inter }}$, the timescale for interadsorbate energy coupling. However, we can set bounds on this value. We expect that top site should couple no more strongly to the electron bath than the bridge site CO; thus we set the lower limit for $\tau_{\mathrm{el} 2}$, the top-site-electron-bath coupling time, to $3.32 \mathrm{ps}$, the coupling time observed for the 0.64-ML adlayer. For an upper limit, we take the case where the top-site $\mathrm{CO}$ is completely decoupled from the substrate electrons. These two limits give interadsorbate coupling times, $\tau_{\text {inter }}$, of 13.3 and 3.2 ps. If the coupling strength were to scale with the square of the fractional occupancy of the $2 \pi^{*}$ orbital, ${ }^{5}$ we would predict value of $\sim 4.7$ ps for $\tau_{\mathrm{el} 2}$, based on the 3.32-ps bridge-site coupling time and the fractional occupancies given by Gajdoš. ${ }^{48}$ This results in our preferred estimate for $\tau_{\text {inter }}$ of $\sim 7 \mathrm{ps.}$

Evidence for coverage-dependent energy transfer rates mediating desorption can be seen in measurements made for $\mathrm{CO}$ fs-PID from $\mathrm{Ru}(001)$ and $\mathrm{Pt}(111)$. In the case of $\mathrm{Ru}(001)$, Funk et al., reported that a twofold increase in the desorption probability and a slight increase in 2PC width (from 19 to $20 \mathrm{ps)} \mathrm{accompanied} \mathrm{an} \mathrm{increase} \mathrm{in} \mathrm{coverage} \mathrm{from} 0.33$ to $0.68 \mathrm{ML}{ }^{23}$ The desorption activation energy in this range decreases from 1.65 to $1.2 \mathrm{eV}$. For adsorbate temperatures in the range of $2000 \mathrm{~K}$, as predicted by empirical friction simulations, ${ }^{23}$ this drop in activation energy would correspond to an increase in probability of 10 times, all else being equal. Because the observed increase is only a factor of two, this, together with the $2 \mathrm{PC}$ results, suggests that for $\mathrm{CO} / \mathrm{Ru}(001)$ the substrate coupling strength is inversely correlated to the molecule-surface bond energy, paralleling the correlation we find for $\mathrm{CO} / \mathrm{Pd}(111)$. That the differences in fs-PID desorption probability and coupling strength are less pronounced in the $\mathrm{Ru}(001)$ case may be 
because: (i) the span of activation energies is smaller, (ii) the coupling timescales are longer, ${ }^{23}$ and (iii) though the site population is not known at the highest coverage, it is proposed that the site preference may change from top to hollow with increasing coverage, ${ }^{84}$ such that the $2 \pi^{*}$ occupation ${ }^{48}$ trend is opposite that in Pd and mitigates the decrease in coupling strength with increasing coverage.

In the case of Pt(111), photoyield measurements revealed a 30\% decrease in desorption probability from 0.15 to $0.50 \mathrm{ML} .{ }^{55}$ This more recent report corrected an initial incorrect interpretation $^{85}$ that surface spectroscopic data indicated a remarkable 5-orders of magnitude increase in desorption probability with coverage between 0.07 and $0.37 \mathrm{ML}{ }^{86}$ The decrease in probability with coverage merits further comment because it runs opposite to what we observe for Pd(111) above 0.33 ML, but in the same direction as the trend we observe below 0.33 ML, which we have not yet addressed. For CO/Pt(111) the activation energy decreases from 1.35 to $1.0 \mathrm{eV}$ between 0.15 to $0.50 \mathrm{ML} .^{87}$ This result parallels our observations between 0.1 and $0.33 \mathrm{ML}$ (Figure 3), where the activation energy decrease from $\sim 1.5$ to $1.3 \mathrm{eV}$ is similar to that for $\mathrm{CO} / \mathrm{Pt}(111)$. All else being equal, we would have predicted an increase in desorption probability with coverage based on our analysis of the higher coverage range, not the approximately fivefold decrease that is actually observed. We speculate that the reason for the reversal of the fs-PID probability coverage dependence in the low coverage range may be a consequence of the increasing lateral mobility of the CO with decreasing coverage below 0.33 ML. Specifically, STM measurements of $\mathrm{CO} / \mathrm{Pd}(111)$ have shown that $(\sqrt{3} \times \sqrt{3}) R 30^{\circ}$ islands nucleate at defect sites and are surrounded by a fluxional sea of CO molecules that only transiently occupy 3 fh sites. ${ }^{44}$ Because these mobile molecules are sampling a variety of adsorption sites as they diffuse, we expect that ultrafast excitation catches them in states resembling bridge-site $\mathrm{CO}$, from which they are more 
easily desorbed, as our analysis of the high-coverage adlayers indicates. In such a scenario, two coverage regimes are identified: below $0.33 \mathrm{ML}$ and above 0.33 ML. Below 0.33 ML there are two classes of molecules: those near the center of the $(\sqrt{3} \times \sqrt{3}) R 30^{\circ}$ islands that are essentially fixed at 3-fh sites, and mobile CO that transiently occupy bridge sites. The later are in a state where they have already climbed part way up the desorption barrier, such that their effective desorption barrier is lowered. In addition, they are less tightly coupled to the substrate electron temperature bath. Thus their fs-PID probabilities approach those of bridge-site CO. As the size of the $(\sqrt{3} \times \sqrt{3}) R 30^{\circ}$ islands grows with coverage, so does the proportion of molecules fixed at 3-fh sites, and thus the ensemble-averaged fs-PID probability approaches that associated with 3-fh adsorption. This explains the decrease in probability with increasing coverage below 0.33 ML. Above 0.33 ML this trend is reversed as the site distribution population shifts from 3-fh to bridge site, as we have already discussed. In the $0.33-0.75 \mathrm{ML}$ range diffusion is not as frequent or as facile because it is limited to motion at domain boundaries or involves cooperative motions of neighboring molecules. The adsorbate distribution, though still fluxional, more closely approximates the idealized picture of molecules fixed at particular lattice sites. In both the low and high coverage regimes the underlying concept is the same: $\eta_{\mathrm{el}}$ and the effective $E_{\mathrm{a}}$ are correlated, and the decrease in $E_{\mathrm{a}}$ prevails over the decrease in $\eta_{\mathrm{el}}$ resulting in a net increase in fs-PID probability.

An alternative explanation for the observed decrease in fs-PID probability with increasing coverage below $0.33 \mathrm{ML}$ is that the relative role of defects increases at lower coverage. Because the adsorbates are preferentially bound near defect sites, ${ }^{44}$ it is conceivable that the more tightlybound molecules are more strongly coupled to the substrate, and that the increased electronic coupling wins out over the increase in desorption activation energy, reversing the fs-PID 
probability coverage dependence trend at low coverage. More detailed measurements, including 2PC measurements, in the low coverage range are needed to test these hypothetical explanations.

Finally, we note that, though our model reproduces our observations quite well and offers significant qualitative insights, important details of the molecular dynamics remain to be addressed. For example, adsorbates heated to $\sim 100$ s of Kelvin for $\sim 100$ s of ps will be displaced from their original binding sites. In addition, energy may be transferred from modes expected to be more strongly coupled to the substrate electronic degrees of freedom (e.g., the frustrated rotation) to those that are not (e.g., the frustrated translation). Such phenomena will change the adsorbate-substrate coupling in a time dependent way, affecting the adsorbate-substrate complex's "memory" of the first pulse, and thus influencing its response to the second pulse in a 2PC experiment. The contributions of these dynamics to the decay times remain, for now, "buried" in the single phenomenological coupling parameter $\eta_{\mathrm{el}}$ that we have used to describe the dynamics. The application of molecular dynamics simulations with electronic frictions (MDEF) ${ }^{6}$, $60,61,78,88$ to the $\mathrm{CO} / \mathrm{Pd}(111)$ system would be a next step to understanding the effects of in-plane molecular motion as well as energy transfer among neighboring molecules and the various vibrational modes. Additional experimental investigations, such as time-resolved surface vibrational spectroscopies ${ }^{4}$, 29, 34, 73, 74 would also be desirable to examine the role of vibrational energy transfer. Based on work done in these areas, we offer some final comments.

The adsorption-site dependent electronic coupling in our simple model neglects adsorbate motion. Nevertheless, previous MDEF investigations of other systems support our analysis as a reasonable starting point. $\mathrm{MDEF}$ of $\mathrm{CO} / \mathrm{Cu}(100)^{88}$ and $\mathrm{O}_{2} / \mathrm{Ag}(110)^{78}$ indicate that though laser heating displaces the adsorbates from their initial bonding sites, their dynamics are linked to their initial adsorption site. In particular, the $\mathrm{CO} / \mathrm{Cu}(100)$ simulations show that in-plane motion is to 
some extent constrained by collisions among neighbors, and that the energy transfer leading to desorption occurs on the subpicosecond timescale, leaving little time for lateral displacement. The $\mathrm{O}_{2} / \mathrm{Ag}(110)$ simulations show that the local electron density at the initial adsorption site has a determinative influence on the dynamics: the relative importance of electronic and phononic coupling, the desorption probability and the fluence dependence of the probability, all depend on the initial adsorption site. In addition, our experimental observation that the first-shot desorption probability at $0.75 \mathrm{ML}$ asymptotically approaches $\sim 0.3$ and not unity with increasing fluence is consistent with desorption only from top sites in the saturated layer, supporting the notion that interpreting our results in terms of the initial binding sites is a reasonable starting point. In-depth analysis of the trajectories in MDEF simulations, as well as extension to coverage-dependent studies would provide insight into how the dynamics of highly-excited molecules are so dramatically impacted by their initial binding site.

Regarding the role of mode coupling in the ultrafast dynamics, we have already noted that a temperature-dependent friction in the empirical friction model may serve as a proxy for heat transfer via mode coupling. That the temperature-dependent friction (Model II) best reproduces our observations may indicate that mode coupling mediates energy transfer from the substrate into the reaction coordinate. ${ }^{59,68,69}$ Evidence from other experiments and theory is mounting that the excitation of the molecule-surface external stretch (ES) that leads to desorption is indirect. The picture that is emerging is complex. Among the molecule surface modes-the ES, the frustrated translation (FR) and the frustrated rotation (FR)—it is thought that the frustrated rotation (FR) couples most strongly to the electron temperature bath. ${ }^{6,69,79,89}$ The FR transfers energy to the FT through anharmonic coupling, ${ }^{68,73}$ and it has been proposed that the ES is excited by interadsorbate collisions that convert parallel momentum to normal momentum. ${ }^{73}$ All of the couplings involved 
are likely to depend on coverage. For example, we expect that the electron-FR coupling will decrease with increasing coverage as occupation of the $2 \pi^{*}$ LUMO decreases with the transition from 3fh- to bridge- to top-site occupation. Furthermore, we would expect the frequency of the FT, and the strength with which it is coupled to the FR and ES, to depend on coverage. Specifically, with increasing coverage the FT mode should stiffen and become more anharmonic, leading to stronger coupling to both the FR and ES modes, increasing the desorption probability. Tight molecular packing at higher coverages has also been suggested to increase the efficiency of conversion of parallel momentum (FT) to normal momentum (ES) ${ }^{73}$ These factors play important roles in the probability enhancements and coupling time variations we observe. Our 2PC measurements do not give mode-specific insight, but they indicate a complexity of the dynamics that provides motivation for theoretical and surface spectroscopic investigations of the energy flow in this and similar systems.

\section{Summary}

In conclusion, we summarize our experimental findings and numerical analysis results:

(1) Femtosecond photoinduced desorption of $\mathrm{CO}$ from $\mathrm{CO} / \mathrm{Pd}(111)$ is driven primarily by electron-mediated energy transfer from the substrate to the admolecules.

(2) The fs-PID probability exhibits a strong dependence on coverage that is much larger than previously observed for any other adlayer/metal system.

(3) Nevertheless, the magnitude of the coverage dependence is far smaller than would be predicted based on the coverage dependence of the desorption activation energy alone.

(4) A coverage-dependent coupling, where the coupling strength decreases with decreasing desorption activation energy is consistent with two-pulse correlation measurements made 
for the $(\sqrt{3} \times \sqrt{3}) \mathrm{R}^{\circ} 0^{\circ}$ and $\mathrm{c}(\sqrt{3} \times 5)$ adlayers and consistent with a site-dependent coupling strength where $\tau_{\text {el }}$ (three-fold hollow) $<\tau_{\text {el }}$ (bridge).

(5) The fs-PID probability from the $(2 \times 2)$ saturated adlayer is approximately one-third that expected based on the relatively short coupling time extracted from 2PC measurements. In addition, this coupling time is shorter than for the lower-coverage $\mathrm{c}(\sqrt{3} \times 5)$ adlayer, counter to our expectation that the coupling strength should decrease with decreasing desorption activation energy.

(6) The relatively low fs-PID probability and shorter coupling time for the $(2 \times 2)$ adlayer can be explained by a site-dependent coupling strength where $\tau_{\text {el }}$ (three-fold hollow) < $\tau_{\text {el }}$ (bridge) $<\tau_{\text {el }}$ (top), accompanied by interadsorbate energy transfer from the 3 fh to top site CO. For the $(2 \times 2)$ adlayer, only the top-site CO desorb, accounting for the observed fs-PID probability. Interadsorbate coupling accounts for the enhanced energy transfer rate. The site-dependent coupling trend correlates with calculated $2 \pi^{*}$ orbital fractional occupation and progressions in the $\mathrm{C}-$-surface distance, the $\mathrm{C}-\mathrm{O}$ bond length, and the $\mathrm{C}-\mathrm{O}$ stretch frequency. We estimate the timescale for the interadsorbate energy transfer to be $\sim 7$ ps.

\section{Acknowledgments}

The authors would like to acknowledge Maite Alducin for helpful conversations. This article is based upon work supported in its entirety by the U.S. Department of Energy, Office of Science, Office of Basic Energy Sciences, Chemical Sciences, Geosciences, \& Biosciences (CSGB) Division, Condensed Phase and Interfacial Molecular Science (CPIMS) Program, under Contract No. DE-SC0012704. 


\section{References}

1. R. Ryberg, Surf. Sci. 114, 627 (1982).

2. J. D. Beckerle, R. R. Cavanagh, M. P. Casassa, E. J. Heilweil, and J. C. Stephenson, J. Chem. Phys. 95, 5403 (1991).

3. $\quad$ M. Morin, N. J. Levinos, and A. L. Harris, J. Chem. Phys. 96, 3950 (1992).

4. J. P. Culver, M. Li, L. G. Jahn, R. M. Hochstrasser, and A. G. Yodh, Chem. Phys. Lett. 214, 431 (1993).

5. $\quad$ B. N. J. Persson and M. Persson, Solid State Commun. 36, 175 (1980).

6. J. C. Tully, M. Gomez, and M. Head-Gordon, J. Vac. Sci. Technol. A 11, 1914 (1993).

7. $\quad$ M. Persson and B. Hellsing, Phys. Rev. Lett. 49 (1982).

8. J. C. Ariyasu, D. L. Mills, K. G. Lloyd, and J. C. Hemminger, Phys. Rev. B 28 (1983).

9. R. R. Cavanagh, D. S. King, J. C. Stephenson, and T. F. Heinz, J. Phys. Chem. 97 (1993).

10. B. Gergen, H. Nienhaus, W. H. Weinberg, and E. W. McFarland, Science 294, 2521 (2001).

11. H. Nienhaus, Surf. Sci. Rep. 45, 1 (2002).

12. E. Hasselbrink, Curr. Opin. Solid State Mater. Sci. 10, 192 (2006).

13. C. Frischkorn and M. Wolf, Chem. Rev. 106, 4207 (2006).

14. A. M. Wodtke, D. Matsiev, and D. J. Auerbach, Prog. Suf. Sci. 83, 167 (2008).

15. H. Arnolds and M. Bonn, Surf. Sci. Rep. 65, 45 (2010).

16. P. Saalfrank, Chem. Rev. 106 (2006).

17. J. C. Tully, Annu. Rev. Phys. Chem. 51 (2000).

18. E. H. G. Backus, A. Eichler, A. W. Kleyn, and M. Bonn, Science 310, 1790 (2005).

19. K. Stépán, J. Güdde, and U. Höfer, Phys. Rev. Lett. 94, 236103 (2005). 
20. J. A. Prybyla, T. F. Heinz, J. A. Misewich, M. M. T. Loy, and J. H. Glownia, Phys. Rev. Lett. 64, 1537 (1990).

21. F. Budde, T. F. Heinz, M. M. T. Loy, J. A. Misewich, F. de Rougemont, and H. Zacharias, Phys. Rev. Lett. 66, 3024 (1991).

22. J. A. Misewich, A. Kalamarides, T. F. Heinz, and M. M. T. Loy, J. Chem. Phys. 100, 736 (1994).

23. S. Funk, M. Bonn, D. N. Denzler, C. Hess, M. Wolf, and G. Ertl, J. Chem. Phys. 112, 9888 (2000).

24. S. Deliwala, R. J. Finlay, J. R. Goldman, T. H. Her, W. D. Mieher, and E. Mazur, Chem. Phys. Lett. 242, 617 (1995).

25. D. G. Busch and W. Ho, Phys. Rev. Lett. 77, 1338 (1996).

26. M. Bonn, S. Funk, C. Hess, D. N. Denzler, C. Stampfl, M. Scheffler, M. Wolf, and G. Ertl, Science 285, 1042 (1999).

27. H. Arnolds, Surf. Sci. 548, 151 (2004).

28. P. Szymanski, A. L. Harris, and N. Camillone, III, J. Phys. Chem. C 112, 15802 (2008).

29. M. Bonn, C. Hess, S. Funk, J. H. Miners, B. N. J. Persson, M. Wolf, and G. Ertl, Phys. Rev. Lett. 84, 4653 (2000).

30. I. M. Lane, D. A. King, Z. P. Liu, and H. Arnolds, Phys. Rev. Lett. 97, 186105 (2006).

31. P. Szymanski, A. L. Harris, and N. Camillone, III, J. Chem. Phys. 126, 214709 (2007).

32. A. Eichler, F. Mittendorfer, and J. Hafner, Phys. Rev. B 62, 4744 (2000).

33. F. Weik, A. de Meijere, and E. Hasselbrink, J. Chem. Phys. 99, 682 (1993).

34. T. Omiya and H. Arnolds, J. Chem. Phys. 141, 214705 (2014).

35. D. P. Woodruff, B. E. Hayden, K. Prince, and A. M. Bradshaw, Surf. Sci. 123, 397 (1982). 
36. M. Tüshaus, W. Berndt, H. Conrad, A. M. Bradshaw, and B. Persson, Appl. Phys. A 51, 91 (1990).

37. F. M. Hoffmann, Surf. Sci. Rep. 3, 107 (1983).

38. W. K. Kuhn, J. Szanyi, and D. W. Goodman, Surf. Sci. 274, L611 (1992).

39. M. Morkel, G. Rupprechter, and H.-J. Freund, J. Chem. Phys. 119, 10853 (2003).

40. H. Ohtani, M. A. Van Hove, and G. A. Somorjai, Surf. Sci. 187, 372 (1987).

41. H. Conrad, G. Ertl, and J. Küppers, Surf. Sci. 76, 323 (1978).

42. T. Gießel, O. Schaff, C. J. Hirschmugl, V. Fernandez, K.-M. Schindler, A. Theobald, S. Bao, R. Lindsay, W. Berndt, A. M. Bradshaw, C. Baddeley, A. F. Lee, R. M. Lambert, and D. P. Woodruff, Surf. Sci. 406, 90 (1998).

43. S. Surnev, M. Sock, M. G. Ramsey, F. P. Netzer, M. Wiklund, M. Borg, and J. N. Andersen, Surf. Sci. 470, 171 (2000).

44. M. K. Rose, T. Mitsui, J. Dunphy, A. Borg, D. F. Ogletree, M. Salmeron, and P. Sautet, Surf. Sci. 512, 48 (2002).

45. D. Loffreda, D. Simon, and P. Sautet, Surf. Sci. 425, 68 (1999).

46. J. Zhang, Z. Wang, and Z. X. Wang, Surf. Interface Anal. 43, 1038 (2011).

47. C. Fan and W.-D. Xiao, Comp. Theo. Chem. 1004, 22 (2013).

48. M. Gajdoš, A. Eichler, and J. Hafner, J. Phys. Condens. Matter 16, 1141 (2004).

49. L. M. Struck, L. J. Richter, S. A. Buntin, R. R. Cavanagh, and J. C. Stephenson, Phys. Rev. Lett. 77, 4576 (1996).

50. P. Szymanski, A. L. Harris, and N. Camillone, III, Surf. Sci. 601, 3335 (2007).

51. H. Arnolds, R. J. Levis, and D. A. King, Chem. Phys. Lett. 380, 444 (2003).

52. J. Gladh, T. Hansson, and H. Öström, Surf. Sci. 615, 65 (2013). 
53. See supplemental material at [URL to be inserted by AIP] for sample LEED images and a tabulation of the $\chi^{2}$ values for the 2PC fits.

54. P. Szymanski, A. L. Harris, and N. Camillone, III, J. Phys. Chem. A 111, 12524 (2007).

55. W. G. Roeterdink, E. H. G. Backus, A. W. Kleyn, and M. Bonn, Phys. Rev. Lett. 93, 249601 (2004).

56. L. Cai, X. Xiao, and M. M. T. Loy, J. Chem. Phys. 115, 9490 (2001).

57. M. Bonn, D. N. Denzler, S. Funk, M. Wolf, S. S. Wellershoff, and J. Hohlfeld, Phys. Rev. B 61, 1101 (2000).

58. D. N. Denzler, C. Frischkorn, C. Hess, M. Wolf, and G. Ertl, Phys. Rev. Lett. 91, 226102 (2003).

59. M. Lawrenz, K. Stépán, J. Güdde, and U. Höfer, Phys. Rev. B 80, 075429 (2009).

60. G. Fuchsel, T. Klamroth, S. Monturet, and P. Saalfrank, Phys. Chem. Chem. Phys. 13, 8659 (2011).

61. A. C. Luntz, M. Persson, S. Wagner, C. Frischkorn, and M. Wolf, J. Chem. Phys. 124, 244702 (2006).

62. S. I. Anisimov, B. L. Kapeliovich, and T. L. Perel'man, Sov. Phys. JETP 39, 375 (1974).

63. F. Budde, T. F. Heinz, A. Kalamarides, M. M. T. Loy, and J. A. Misewich, Surf. Sci. 283, 143 (1993).

64. S. L. Tait, Z. Dohnálek, C. T. Campbell, and B. D. Kay, J. Chem. Phys. 122, 164707 (2005).

65. K. Stépán, M. Dürr, J. Güdde, and U. Höfer, Surf. Sci. 593, 54 (2005).

66. J. Güdde and U. Höfer, J. Phys.: Condens. Matter 18, S1409 (2006).

67. M. Brandbyge, P. Hedegård, T. F. Heinz, J. A. Misewich, and D. M. Newns, Phys. Rev. B 52, 6042 (1995). 
68. M. Hayashi, Y. Ootsuka, M. Paulsson, B. N. J. Persson, and H. Ueba, Phys. Rev. B 80, 245409 (2009).

69. H. Ueba and Y. Ootsuka, Phys. Rev. B 87, 205403 (2013).

70. M. V. Pykhtin, S. P. Lewis, E. J. Mele, and A. M. Rappe, Phys. Rev. Lett. 81, 5940 (1998).

71. C. J. Hirschmugl, G. P. Williams, F. M. Hoffmann, and Y. J. Chabal, Phys. Rev. Lett. 65, 480 (1990).

72. F. Hofmann and J. P. Toennies, Chem. Rev. 96, 1307 (1996).

73. K.-i. Inoue, K. Watanabe, and Y. Matsumoto, J. Chem. Phys. 137, 024704 (2012).

74. T. A. Germer, J. C. Stephenson, E. J. Heilweil, and R. R. Cavanagh, J. Chem. Phys. 101, 1704 (1994).

75. A. Graham, F. Hofmann, and J. P. Toennies, J. Chem. Phys. 104, 5311 (1996).

76. S. P. Lewis and A. M. Rappe, Mat. Res. Soc. Symp. Proc. 408, 391 (1996).

77. H. Kato, H. Okuyama, S. Ichihara, M. Kawai, and J. Yoshinobu, J. Chem. Phys. 112, 1925 (2000).

78. I. Lončarić, M. Alducin, P. Saalfrank, and J. I. Juaristi, Phys. Rev. B 93, 014301 (2016).

79. M. Head-Gordon and J. C. Tully, Phys. Rev. B 46, 1853 (1992).

80. B. O. Hall and H. M. James, Phys. Rev. B 13, 3590 (1976).

81. A. Bondi, J. Phys. Chem. 68, 441 (1964).

82. H. Kato, M. Kawai, and J. Yoshinobu, Phys. Rev. Lett. 82 (1999).

83. F. Christian, J. Phys. Condens. Matter 20, 313002 (2008).

84. S. H. Payne, J. S. McEwen, H. J. Kreuzer, and D. Menzel, Surf. Sci. 594, 240 (2005).

85. F. Fournier, W. Zheng, S. Carrez, H. Dubost, and B. Bourguignon, Phys. Rev. Lett. 93, 249602 (2004). 
86. F. Fournier, W. Zheng, S. Carrez, H. Dubost, and B. Bourguignon, Phys. Rev. Lett. 92, 216102 (2004).

87. G. Ertl, M. Neumann, and K. M. Streit, Surf. Sci. 64, 393 (1977).

88. C. Springer and M. Head-Gordon, Chem. Phys. 205, 73 (1996).

89. $\quad$ N. Lorente and H. Ueba, Eur. Phys. J. D 35, 341 (2005). 